\title{
Influence of the Capping Ligand on the Band Gap and Electronic Levels of PbS Nanoparticles through Surface Atomistic Arrangement Determination
}

\author{
Diana Fabiola Garcia-Gutierrez, ${ }^{\dagger, \ddagger}$ Laura Patricia Hernandez-Casillas, ${ }^{\dagger \neq}$ Maria Victoria Cappellari, ${ }^{\S}$

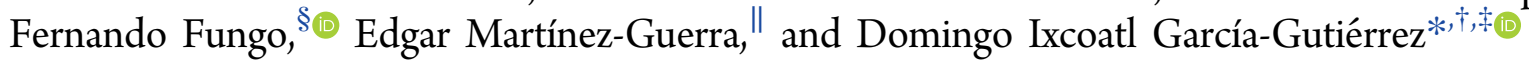

${ }^{\dagger}$ Facultad de Ingeniería Mecánica y Eléctrica, FIME and "Facultad de Ciencias Físico Matemáticas, FCFM, Universidad Autónoma de Nuevo León, UANL, Av. Universidad S/N, Cd. Universitaria, San Nicolás de los Garza, C.P. 66450 Nuevo León, Mexico

${ }^{\ddagger}$ Centro de Innovación, Investigación y Desarrollo en Ingeniería y Tecnología, CIIDIT, Universidad Autónoma de Nuevo León, UANL, Km. 10 de la nueva carretera al Aeropuerto Internacional de Monterrey, PIIT Monterrey, Apodaca, C.P. 66600 Nuevo León, Mexico

${ }^{\S}$ Departamento de Química, Universidad Nacional de Rio Cuarto, UNRC. CONICET, RN36 601, X5804BYA Río Cuarto, Córdoba, Argentina

\section{Supporting Information}

ABSTRACT: Lead sulfide ( $\mathrm{PbS}$ ) nanoparticles were synthesized by chemical methods with different sizes and different capping ligands (oleic acid, myristic acid, and hexanoic acid), avoiding ligand exchange procedures, to study the effect of characteristics of the capping ligands on their energy levels and band gap values. Experimental results (UV-vis-NIR, Fourier transform infrared, and Raman spectroscopies, cyclic voltammetry, transmission electron microscopy, and electron energy loss spectroscopy) showed a marked influence of the capping ligand nature on the electro-optical properties of $\mathrm{PbS}$ nanoparticles with a very similar size. Differences were observed in the atomistic arrangement on the nanoparticle surface and phonon vibrations with the different capping ligands. These observations suggest that the electrooptical properties of $\mathrm{PbS}$ nanoparticles are not only determined by their size, through quantum confinement effects, but also strongly affected by the atomistic arrangement on the nanoparticle surface, which is determined by the capping ligand nature.

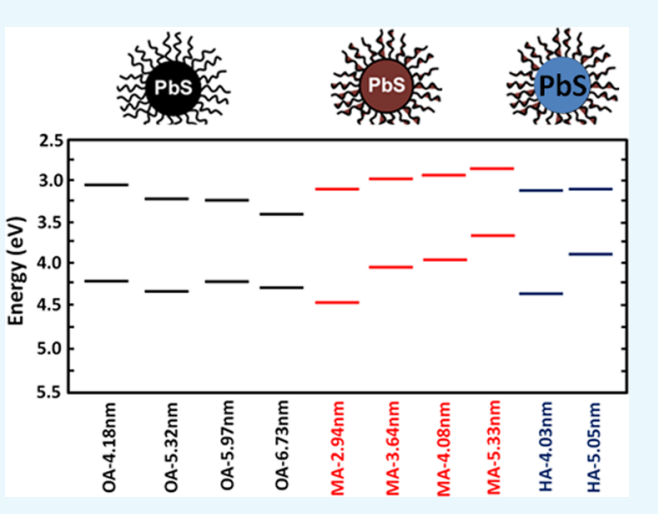

\section{INTRODUCTION}

In recent years, lead sulfide nanoparticles have become promising building blocks for optoelectronic devices, mainly because of the fact that their absorbance and emission can be tailored by means of controlling their size. Their optical band gap can be tuned between 0.5 and $1.5 \mathrm{eV}$, with a photoresponse in the near-infrared, characteristics that make them attractive for applications in photodetectors ${ }^{1-3}$ and photovoltaic devices. ${ }^{4-6}$ Recent reports on photovoltaic devices based on $\mathrm{PbS}$ nanoparticles have focused on the concept of "band alignment engineering", ${ }^{7-9}$ which basically implies the tuning of the energy levels of the nanoparticles by varying their surface ligands. This action not only allows certain control on the value of the energy levels of the nanoparticles but also makes them more stable under atmospheric conditions. Through this concept, highly efficient solar cells have been fabricated based on $\mathrm{PbS}$ nanoparticles, with efficiencies over 9\%. The effect of the surface ligand of nanoparticles on the values of the highest occupied molecular orbital (HOMO) level and lowest unoccupied molecular orbital (LUMO) level has recently been reported. ${ }^{10-12}$ Previously, variations in the energy levels of semiconductor nanoparticles were attributed mainly to quantum confinement effects related to the variations in nanoparticle size; these variations in the energy levels were evidenced through the increment of the optical band gap energy $\left(E_{\mathrm{g}}^{\mathrm{opt}}\right)$, corresponding to a decrement in the nanoparticle size, and they were measured via UV-vis-NIR optical absorbance spectroscopy. ${ }^{11,13}$ Nonetheless, Jasieniak et al. ${ }^{11}$ were able to measure, through photoelectron spectroscopy in air, that for the same CdSe nanoparticle size $(4.7 \mathrm{~nm})$, the variation of the surface ligand had a marked effect on the ionization energy (IE). For the case of alkyl amines of different chain lengths, the IE observed was around $5.4 \mathrm{eV}$, regardless of the length of the chain, whereas for the cases of trioctylphosphine (TOP) oxide, oleic acid (OA), alkane thiols, and pyridine, the IEs measured were 5.45, 5.60, 5.65, and 5.75 $\mathrm{eV}$, respectively. This finding showed a clear impact of the surface ligand of nanoparticles on the IE measured. In a similar

Received: October 11, 2017

Accepted: December 29, 2017

Published: January 11, 2018 
study, Brown et al., ${ }^{10}$ using ultraviolet photoelectron spectroscopy, were able to measure the energy level shifts of $\mathrm{PbS}$ nanoparticles treated with 12 different ligands; they measured valence band (VB) maxima varying within a range of $0.9 \mathrm{eV}$ for nanoparticles of the same size just by modifying the surface ligand through a "solid-state ligand exchange" method. The observed energy levels shifts were attributed to the contribution from both the nanoparticle/ligand interface dipole ${ }^{12}$ and the intrinsic dipole moment of the ligand molecule itself. ${ }^{14}$ On the other hand, Soreni-Harari et al. ${ }^{12}$ observed the same trend when the ligand molecule is changed; however, they did so by using cyclic voltammetry (CV) and scanning tunneling spectroscopy. Using $\mathrm{CV}$, they were able to observe the energy level tuning, following the ligand exchange, by determining the HOMO level by analyzing nonaqueous solutions of the nanoparticles. In their studies, they focused on determining the HOMO level because $E_{\mathrm{g}}^{\text {opt }}$ did not show any variation after the ligand exchange; thus, a corresponding shift was expected in the LUMO level. It has been reported in the literature that semiconductor nanoparticles after a ligand exchange process show small variations (tens of nanometers), or no variations at all, in their first absorption peak, compared to the original capping ligand; thus, their $E_{\mathrm{g}}^{\text {opt }}$ remains basically the same., ${ }^{72,14}$

However, for the case of $\mathrm{PbS}$ nanoparticles, when the literature is reviewed in detail, reports can be found where similar synthesis methodologies produce nanoparticles with the same measured size that show differences in their first absorption peak maxima higher than $100 \mathrm{~nm}$. Debellis et al. ${ }^{15}$ reported the synthesis of $\mathrm{PbS}$ nanoparticles of 2.9, 3.5, 4.9, and $6.7 \mathrm{~nm}$ in size by the "one-pot" method, with their first absorption peak maxima at approximately $870,964,1339$, and $1723 \mathrm{~nm}$, respectively. On the other hand, Hyun et al., ${ }^{16}$ using the same synthesis methodology, reported $\mathrm{PbS}$ nanoparticles of $2.9,3.4,4.8$, and $6.6 \mathrm{~nm}$ in size, with their first absorption peak maxima at approximately 730, 870, 1245, and $1690 \mathrm{~nm}$, respectively. The differences in the first absorption peak maxima for $\mathrm{PbS}$ nanoparticles of 2.9, 3.5, and $4.9 \mathrm{~nm}$ in size between these two studies are of 140, 94, and $94 \mathrm{~nm}$, respectively. In both studies, the capping ligand is based on a lead oleate layer found on the surface of the nanoparticles, which is formed during the growth process of the $\mathrm{PbS}$ nanoparticles, as it has been previously reported for lead chalcogenide nanoparticles when synthesized by the one-pot method. ${ }^{17,18}$ From these observations, it is implied that the nanoparticle size is not the only parameter determining the first absorption peak maximum value in semiconductor nanoparticles and thus $E_{\mathrm{g}}^{\mathrm{opt}}$. One small difference between the two synthesis methodologies, even though both $\mathrm{PbS}$ nanoparticles were synthesized using the one-pot method, was the fact that Hyun et al. suggest the use of TOP, as the dilution solvent of the $S$ precursor, bis(trimethylsilyl)sulfide (TMS), as well as 1octadecene (ODE), whereas Debellis et al. only specify the use of ODE. Hines and Scholes ${ }^{13}$ have reported the use of both TOP and ODE, as the dilution solvent for TMS, claiming no perceivable effect on the synthesized $\mathrm{PbS}$ nanoparticles. However, this small difference in the synthesis process could be the reason for the clear differences in the optical absorbance of $\mathrm{PbS}$ nanoparticles with the same size.

Similar observations have been made in magic-sized clusters (MSCs) of some semiconductor materials, where absorption peak maximum shifts have been observed after ligand exchange procedures are performed. ${ }^{19-23}$ Possible explanations for this phenomenon that have been proposed in the literature include an atomic surface rearrangement on the MSCs or even a phase change ${ }^{23,24}$ however, it has been reported that this phenomenon is observed only in MSCs, not in nanoparticles, and only after performing ligand exchange procedures.

On the other hand, it has been reported for PbSe nanoparticles that varying the ratio between the capping ligand and the $\mathrm{Pb}$ precursor has an impact on the final shape and facets found on the surface of the synthesized nanoparticles. ${ }^{25}$ This variation of the capping ligand concentration found on the reaction affected the degree of surface coverage on the different facets displayed by $\mathrm{PbSe}$ nanoparticles, thus affecting the surface energy of each facet. The variation of the surface energies of the different facets found on the surface of the $\mathrm{PbSe}$ nanoparticles ( $\{100\}$ and $\{111\}$ facets) with ligand coverage determines the equilibrium core shape of the $\mathrm{PbSe}$ nanoparticles. The equilibrium shape of a crystal is given by the surface energies of the facets and is obtained through the Wulff construction. ${ }^{26}$ Theoretical studies for $\mathrm{PbS}$ nanoparticles have reported that varying the type of capping ligand (lead oleate, formate, acetate, nonanoate, trans-oleate, and cis-oleate) has an effect on the surface energy of the different facets found on the surface of face-centered cubic (fcc) $\mathrm{PbS}$ nanoparticles ( $\{100\}$ and $\{111\}$ ), thus affecting the final shape of the nanoparticles and determining the atomistic arrangement on their surface. ${ }^{27}$ None of the previous studies report on the possible effects of such a nanoparticle shape or atomistic surface arrangement of nanoparticles on their optical properties or electronic structure.

In the present work, $\mathrm{PbS}$ nanoparticles were synthesized by chemical methods, through the one-pot method, using different capping ligands OA, myristic acid (MA), and hexanoic acid (HA), in which capping ligand exchange procedures are avoided. Afterward, the energy levels of the synthesized PbS nanoparticles were measured from UV-vis-NIR absorbance spectroscopy, CV measurements of the nanoparticles in solution, and electron energy loss spectroscopy (EELS) measurements. Additionally, the synthesized $\mathrm{PbS}$ nanoparticles were thoroughly characterized by transmission electron microscopy (TEM) and its related techniques, along with Fourier transform infrared (FTIR), and Raman spectroscopy measurements. Once the morphological and physicochemical characteristics of the nanoparticles were determined, a correlation of the nanoparticle size and the capping ligand characteristic with the energy level behavior was made. A clear effect of the nanoparticle size on the calculated values for the energy levels was observed, as reported previously and expected from quantum confinement effects. However, the variation of the molecular characteristics of the capping ligand not only affected the final values of the HOMO and LUMO levels but also had an effect on the value of $E_{\mathrm{g}}^{\text {opt }}$ because nanoparticle systems with different capping ligands and very similar sizes and size distribution displayed optical energy band gaps with marked differences, showing variations only in the atomistic arrangement on their surface, an aspect that has not been deeply analyzed in the literature.

\section{EXPERIMENTAL SECTION}

2.1. Materials. Lead oxide ( $\mathrm{PbO}, 99.999 \%$, Aldrich), ODE (90\%, Aldrich), diphenylphosphine (DPP, 98\%, Aldrich), TMS (synthesis grade, Aldrich), HA (99\%, Aldrich), MA (98\%, Fluka), OA (90\%, Aldrich), tetrahydrofuran (THF), tetrabutylammonium perchlorate (TBAP), tetrabutylammonium hexafluorophosphate, anhydrous toluene, anhydrous hexane, anhydrous chloroform, anhydrous ethanol, and anhydrous acetone 

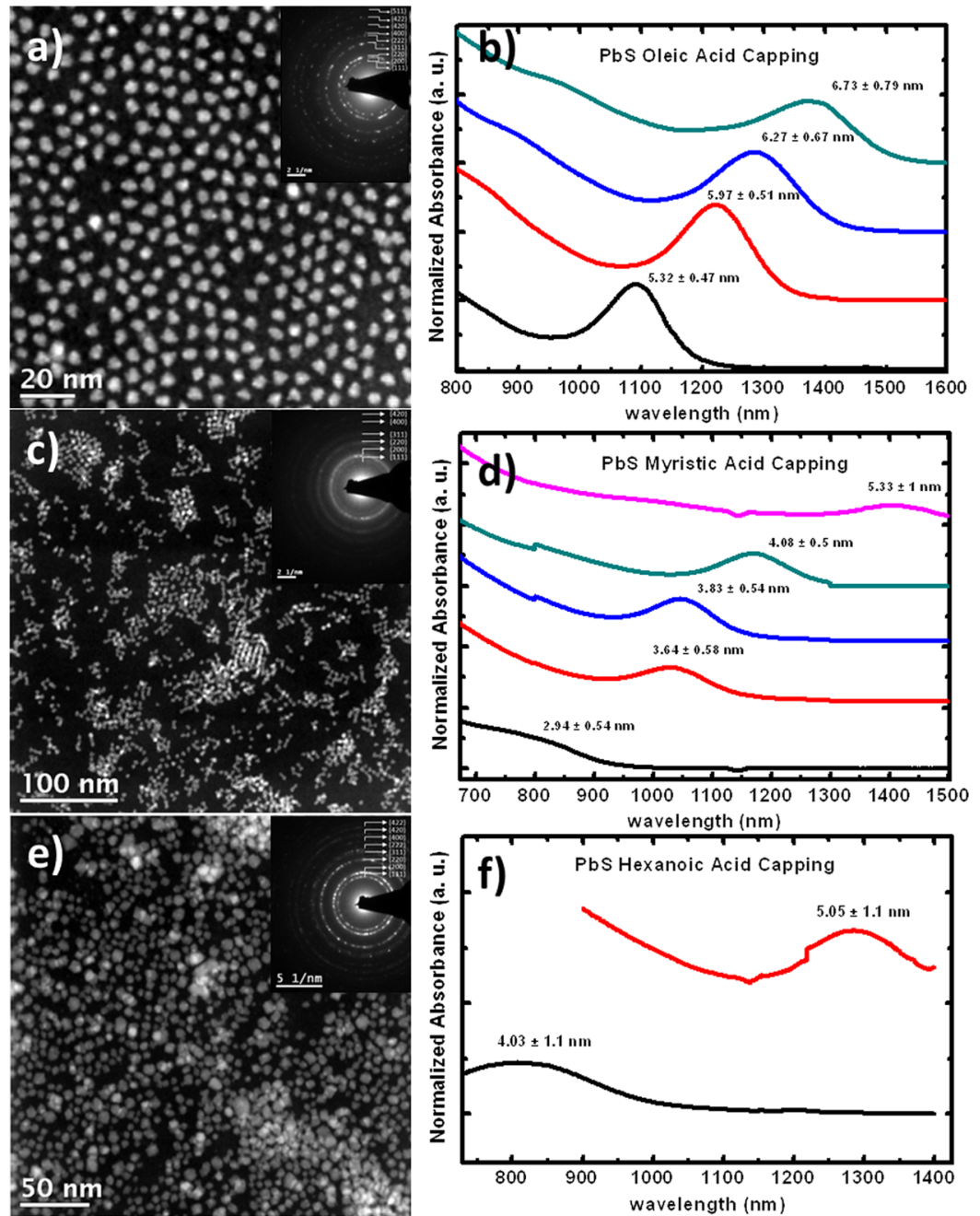

Figure 1. HAADF-STEM images corresponding to (a) OA-, (c) MA-, and (e) HA-capped PbS nanoparticles. Insets: representative SAED patterns for the nanoparticle systems. On the left side, UV-vis-NIR absorption spectra showing a size-dependent behavior of (b) PbS-OA-capped, (d) PbSMA-capped, and (f) PbS-HA-capped nanoparticles. Average size and standard deviation for each studied sample are included.

were purchased from Sigma-Aldrich, whereas dissolvents such as anhydrous methanol, chloroform, hexane, acetone, methanol, and ethanol were purchased from J.T. Baker and CTR Scientific. All reagents were used as received without further purification, except for THF.

2.2. Synthesis of PbS Nanoparticles with Different Capping Ligands. $\mathrm{PbS}$ nanoparticles were synthesized using the slightly modified one-pot synthesis method. The variation of the capping ligand was performed since the preparation of the lead precursor. Three carboxylic acids (CAs) were used to prepare the lead precursor: HA, MA, and OA. First, in a threeneck round-bottom flask, $23 \mathrm{~mL}$ of ODE, $0.45 \mathrm{~g}$ of $\mathrm{PbO}$, and the $\mathrm{CA}$ in amounts reaching a ratio of $2.5 \mathrm{CA} / 1 \mathrm{~Pb}$ were mixed and heated at $150{ }^{\circ} \mathrm{C}$ for $1 \mathrm{~h}$. For the sulfur precursor, $2 \mathrm{~mL}$ of ODE, $0.2 \mathrm{~mL}$ of TMS, and $0.1 \mathrm{~mL}$ of DPP were stirred for 30 min at room temperature in a glovebox under a nitrogen atmosphere. For the synthesis of the $\mathrm{PbS}$ nanoparticles, the sulfur precursor was quickly added with a syringe into the lead precursor, and the nucleation and growth of the nanoparticles occurred immediately. The colorless lead precursor turned dark at the moment the sulfur precursor was added. Different reaction times $(10,120,300$, and $600 \mathrm{~s})$ were used to vary the nanoparticle size. The synthesized $\mathrm{PbS}$ nanoparticles were separated from the organic byproducts by centrifugation with an excess of acetone and then redispersed in anhydrous toluene; this cleaning process was repeated five more times for $\mathrm{PbS}-\mathrm{OA}$, three times for PbS-MA, and only once for PbS-HA nanoparticles. More details about the synthesis of the $\mathrm{PbS}$ nanoparticles with different capping ligands can be found in ref 28.

2.3. Characterization. The synthesized nanoparticles were characterized by means of TEM with the use of an FEI TEM Titan $\mathrm{G}^{2}$ 80-300 operated at $300 \mathrm{kV}$, with scanning transmission electron microscopy (STEM) capabilities, and equipped with a high-angle annular dark-field (HAADF) detector from Fishione; a bright-field STEM detector from Gatan; an ADF STEM detector from Gatan; and an EDAX energy-dispersive X-ray spectroscopy (EDXS) detector. Aberration-corrected STEM images along with EELS spectra were acquired in a TEM JEOL JEM-ARM 200F equipped with a Gatan GIF Tridiem filter; all EELS spectra were acquired with an accelerating voltage of $80 \mathrm{kV}$ and a collection angle $(\beta)$ of $2.1 \mathrm{mrad}$; and these working conditions produced a zero-loss peak with an fwhm of $0.7 \mathrm{eV}$. The TEM samples were prepared as follows: one drop of a solution containing the nanoparticles was cast on a carbon-coated copper grid. A UV-vis-NIR optical absorption spectrometer Cary 5000 from Agilent was used to acquire the absorption spectra from the nanoparticles. 

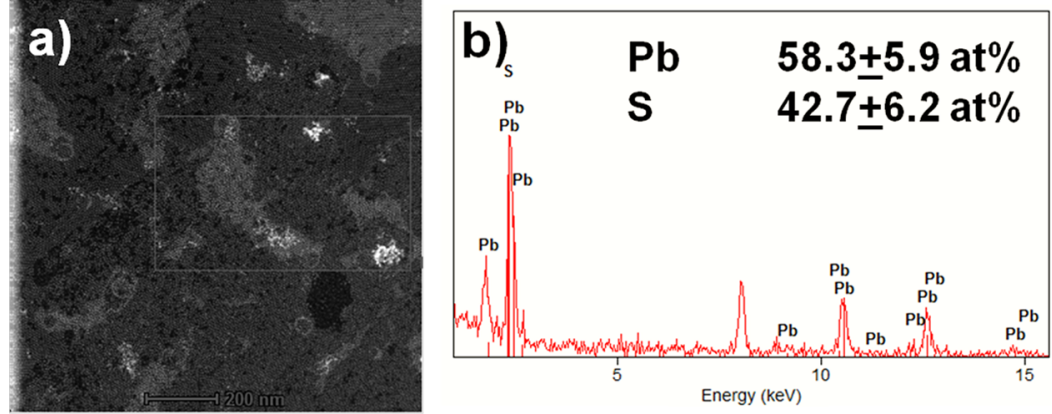

Figure 2. (a) Low-magnification HAADF-STEM image of the nanoparticles, the gray rectangle showing the area analyzed. (b) Representative EDXS spectrum for the $\mathrm{PbS}$ nanoparticles synthesized with $\mathrm{OA}$ as the capping ligand.

The surface chemistry of the $\mathrm{PbS}$ nanoparticles was evaluated through FTIR spectroscopy studies in the transmission mode using a Thermo Fisher Nicolet 6770 FT-IR spectrometer, with a detection range between 400 and $4000 \mathrm{~cm}^{-1}$ and a resolution of $4 \mathrm{~cm}^{-1}$ with 32 scans per sample. Raman spectra were acquired with a Thermo Fisher DXR Raman microscope using a laser excitation of $532 \mathrm{~nm}$, a laser power of $7 \mathrm{~mW}$, a rectangular aperture of $50 \mu \mathrm{m}$, and an objective lens of $10 \times$. Raman studies were performed on thin films of the $\mathrm{PbS}$ nanoparticles deposited by drop casting on glass substrates.

2.4. Electrochemical Studies. Electrochemical studies were carried out with an Interface 1000 potentiostat/ galvanostat/ZRA (Gamry Instruments) in a three-electrode cell. A platinum $(\mathrm{Pt})$ disc was used as the working electrode $\left(2.01 \mathrm{~mm}^{2}\right)$, a silver wire was used as the reference electrode, and a Pt coil was used as the counter electrode. The Pt working electrode was polished with $0.05 \mu \mathrm{m} \mathrm{Al}_{2} \mathrm{O}_{3}$ and then sonicated in deionized water. Before using the polished Pt electrode, cleanliness of the metal surface was checked by obtaining the characteristic voltammogram of $\mathrm{Pt}$ in $0.1 \mathrm{M} \mathrm{H}_{2} \mathrm{SO}_{4} . \mathrm{PbS}$ nanoparticle solutions were prepared in freshly distilled THF with $0.1 \mathrm{M}$ TBAP as the supporting electrolyte and then deaerated with high-purity Ar. Before each experiment, electrolyte blank was made to discard possible electrochemically active interferences. All potential values reported are expressed relative to ferrocene/ferrocenium redox couple $(\mathrm{Fc} /$ $\mathrm{Fc}^{+}=0.40 \mathrm{~V}$ vs SCE), which was used as an internal standard. ${ }^{29}$ All reported voltammograms were acquired with a velocity of $100 \mathrm{mV} / \mathrm{s}$.

\section{RESULTS AND DISCUSSION}

$\mathrm{PbS}$ nanoparticles synthesized with $\mathrm{HA}$ (PbS-HA) showed a quasi-spherical morphology with a high size distribution (approximately $1.1 \mathrm{~nm}$ ); at short reaction times, no clear agglomerates could be observed (Figure S1). However, at a reaction time of $300 \mathrm{~s}$ and higher, coalescence of nanoparticles became evident, originating nanoparticles of $10 \mathrm{~nm}$ in size or larger, which formed agglomerates with a size larger than 100 $\mathrm{nm}$. Observations of the $\mathrm{PbS}$ nanoparticles synthesized with MA (PbS-MA) showed a system with good size distribution (between 0.5 and $1 \mathrm{~nm}$ ), with average nanoparticle sizes ranging from 2.9 to $5.3 \mathrm{~nm}$, corresponding to the different reaction times. No nanoparticle agglomerates could be observed at any reaction time, nor the evidence of coalescence between nanoparticles (Figure S2). PbS nanoparticles synthesized with $\mathrm{OA}$ ( $\mathrm{PbS}-\mathrm{OA})$ showed the lowest size distribution (between 0.47 and $0.79 \mathrm{~nm}$ ), with average nanoparticle sizes ranging from 5.3 to $6.7 \mathrm{~nm}$, corresponding to the different reaction times. Similar to the MA case, no nanoparticle agglomerates were observed at any reaction time, nor the evidence of coalescence was observed (Figure S3). For the determination of the average size and standard deviation displayed by the $\mathrm{PbS}$ nanoparticles synthesized at the different reaction times, 300 nanoparticles were measured.

Figure 1a,c,e show the typical STEM-HAADF images of the synthesized $\mathrm{PbS}$ nanoparticles with different capping ligands. It can be observed that as the $\mathrm{C}$ chain length gets shorter in the capping ligand, the size dispersion increases. The $\mathrm{PbS}$ nanoparticles synthesized with OA were highly monodisperse with a low standard deviation in size (Figure 1a), whereas the $\mathrm{PbS}$ nanoparticles synthesized with HA were more polydisperse with higher standard deviations (approximately $1.1 \mathrm{~nm}$ ) (Figure 1e). Shown as insets are typical selected area electron diffraction (SAED) patterns from the synthesized PbS nanoparticles, where the interplanar distances identified correspond to the planes $\{111\},\{200\},\{220\},\{311\},\{222\}$, $\{400\},\{420\},\{422\}$, and $\{511\}$ of the fcc crystal structure (JCPDS 77-0244) of PbS. All SAED patterns from the synthesized $\mathrm{PbS}$ nanoparticles, regardless of the capping ligand used in their synthesis, showed lattice spacings corresponding to this fcc crystal structure.

Different absorption UV-vis-NIR spectra acquired for the synthesized samples are shown in Figure 1b,d,f. Clear quantum confinement effects can be observed as the size of the nanoparticles decreases. For the case of the PbS-OA nanoparticles, the first absorption peak maximum ranged between 1095 and $1368 \mathrm{~nm}$ (Figure 1b). In the case of the PbS-MA nanoparticles, the range of the first absorption peak maximum was observed between 779 and $1400 \mathrm{~nm}$ (Figure 1d), whereas for the PbS-HA nanoparticles, only two reaction times produce a well-defined absorption peak; these two values were 817 and $1285 \mathrm{~nm}$ (Figure 1f), corresponding to the average sizes of 4.03 and $5.05 \mathrm{~nm}$, respectively.

The composition of the synthesized $\mathrm{PbS}$ nanoparticles with different capping ligands was determined by means of EDXS studies. Figure 2 shows the typical EDXS spectrum for the synthesized $\mathrm{PbS}$ nanoparticles capped with $\mathrm{OA}$. As previously reported for lead chalcogenide nanoparticles synthesized by the one-pot method, ${ }^{17,18,30}$ the current synthesized $\mathrm{PbS}$ nanoparticles also showed a higher concentration of $\mathrm{Pb}$ compared to $\mathrm{S}$. The excess of $\mathrm{Pb}$ on the nanoparticle surface is attributed to the oxidation of $\mathrm{Pb}$ by the capping agent, in this case, OA, MA, and HA, producing a capping layer of the corresponding lead carboxylate (lead oleate, lead myristate, and lead hexanate). This composition trend was similar in all capping ligands used (Figure S4). Table 1 shows the compositions measured for the 
three different capping ligands and the $\mathrm{Pb} / \mathrm{S}$ ratios they showed.

Table 1. Compositions of the Nanoparticles Synthesized with the Three Different Capping Ligands (OA, HA, and $\mathrm{MA})$ and Their Corresponding $\mathrm{Pb} / \mathrm{S}$ Ratios

$\begin{array}{cccc}\text { sample } & \mathrm{Pb} \text { at. } \% & \mathrm{~S} \text { at. } \% & \mathrm{~Pb} / \mathrm{S} \\ \mathrm{PbS}-\mathrm{OA} & 57.3 \pm 5.9 & 42.7 \pm 6.2 & 1.34 \\ \mathrm{PbS}-\mathrm{HA} & 56.3 \pm 5.1 & 43.7 \pm 5.0 & 1.29 \\ \mathrm{PbS}-\mathrm{MA} & 55.3 \pm 6.7 & 44.7 \pm 5.7 & 1.23\end{array}$

The FTIR spectra of the $\mathrm{PbS}$ nanoparticles capped with $\mathrm{OA}$, MA, and HA (Figure S5) showed similar features; all of them displayed bands related to the $\mathrm{C}-\mathrm{H}$ stretching vibration at $\sim 2925 \mathrm{~cm}^{-1}$ due to the asymmetric stretching of the methylene group and at $\sim 2854 \mathrm{~cm}^{-1}$ associated with the symmetric stretching of the methylene group. However, these signals are clearly less intense in the PbS-HA sample, a behavior attributed mainly to the shorter $\mathrm{C}$ chain of HA compared to those of OA and MA. Also, a band can be detected at $1884 \mathrm{~cm}^{-1}$ related to the symmetric stretching of the $\mathrm{C}-\mathrm{H}$ group, which is observed to be less intense in the case of PbS-MA. Another signal can be observed at $\sim 1765 \mathrm{~cm}^{-1}$ in all three samples, associated with the stretching vibration of the $\mathrm{C}=\mathrm{O}$ functional groups (present in the original CA); again, this band showed the lowest intensity for the case of the PbS-MA sample. On the other hand, the bands observed at $\sim 1572 \mathrm{~cm}^{-1}$ in PbS-HA, at $\sim 1515 \mathrm{~cm}^{-1}$ in PbS-MA, and at $1545 \mathrm{~cm}^{-1}$ in PbS-OA samples can be associated with the asymmetric vibration of the ionized carboxyl (COO-), whereas the band at $\sim 1466 \mathrm{~cm}^{-1}$ in $\mathrm{PbS}-\mathrm{HA}$ and PbS-MA and the band at $\sim 1457 \mathrm{~cm}^{-1}$ in the PbSOA sample can be associated with the symmetric vibration of the ionized carboxyl (COO-). The wavenumber separation, $\Delta$, between the asymmetric and symmetric vibrations of the COO- IR bands can be used to determine the type of interaction between the carboxylate group and the metal atom. A larger $\Delta\left(200-320 \mathrm{~cm}^{-1}\right)$ corresponds to a monodentate interaction, whereas a smaller $\Delta$ value $\left(<110 \mathrm{~cm}^{-1}\right)$ is associated with a chelating bidentate interaction. ${ }^{31,32}$ In these results, the value for $\Delta$ obtained is always smaller than 110 $\mathrm{cm}^{-1}$; thus, in all three samples, the capping ligand seems to show a chelating bidentate interaction with the $\mathrm{PbS}$ nanoparticle surface. These results support the idea of the presence of a carboxylate on the surface of the nanoparticles, in this case, lead oleate, myristate, and hexanate. ${ }^{18,31,32}$

Figure 3 shows the Raman spectra of the $\mathrm{PbS}$ nanoparticles synthesized with OA, MA, and HA. The three samples show very similar signals. The bands observed at 135 and $964 \mathrm{~cm}^{-1}$ are related to the photo-oxidation of $\mathrm{PbS}$ by the effect of the laser used for the Raman study and to the formation of $\mathrm{PbO}$ and $\mathrm{PbSO}_{4}$, respectively. ${ }^{33}$ The bands below 80 and around 270 $\mathrm{cm}^{-1}$ are related to the formation of phonons in the crystal lattice of the $\mathrm{PbS}$ nanoparticles. ${ }^{34}$ Bozyigit et al. correlate a band at around $84 \mathrm{~cm}^{-1}$ with the excitation of a longitudinal acoustic (LA) phonon in bulk PbS; they also report how this excitation shifts toward lower wavenumbers for $\mathrm{PbS}$ nanoparticles of between 1 and $8 \mathrm{~nm}$ in size. ${ }^{34}$ For our synthesized $\mathrm{PbS}$ nanoparticles, the band related to the excitation of this LA phonon can be observed at $\sim 78, \sim 76$, and $\sim 76 \mathrm{~cm}^{-1}$ for the nanoparticles synthesized with OA, MA, and HA, respectively. Additionally, the band observed at $\sim 268 \mathrm{~cm}^{-1}$ is related to the formation of a discrete optical phonon on the surface of the

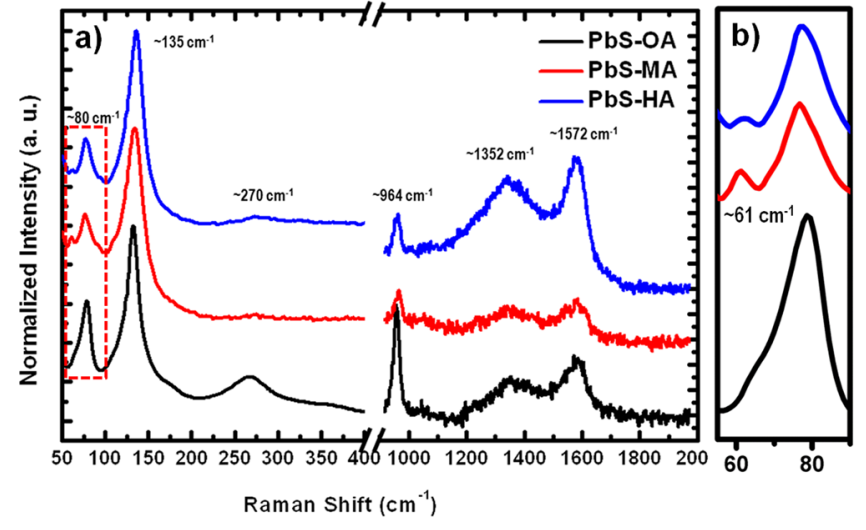

Figure 3. (a) Raman spectra of $\mathrm{PbS}$ nanoparticles with different capping ligands. (b) Zoom-in view of the region marked by the red dotted rectangle in (a).

$\mathrm{PbS}$ nanoparticles, due to the presence of the capping layer, and the discontinuity of the crystal lattice; ${ }^{34}$ this signal is more clearly observed in PbS-OA (Figure 3a). Figure $3 \mathrm{~b}$ shows a zoom-in view of the region marked by the red dotted rectangle in Figure 3a, where an additional band can be clearly observed at $\sim 61 \mathrm{~cm}^{-1}$ in $\mathrm{PbS}-\mathrm{MA}$ and, with much lower intensity, in $\mathrm{PbS}-\mathrm{HA}$, whereas for the case of PbS-OA, this band cannot be clearly observed. The appearance of this band at $\sim 61 \mathrm{~cm}^{-1}$ can be attributed to the "softening" of the nanoparticle surface, proposed by Bozyigit et al. for PbS nanoparticles, ${ }^{34}$ and they also proposed how this softening enables the appearance of a large number $(>100)$ of low-frequency phonon modes. Finally, the bands observed at 1352 and $1580 \mathrm{~cm}^{-1}$ are related to the signals associated with the $\mathrm{C}-\mathrm{C}$ bonds, where the ratio of the signals, the value of the band maximum, and the width of the band indicate the existing ratio between the $\mathrm{sp}^{2}$ and $\mathrm{sp}^{3}$ hybridizations displayed by the $\mathrm{C}$ atoms in the analyzed sample. ${ }^{35}$ In the samples studied, the characteristics of these bands indicate a high degree of disorder displayed by the $\mathrm{C}$ atoms found in the capping layer of the $\mathrm{PbS}$ nanoparticles.

The electrochemical response of the $\mathrm{PbS}$ nanoparticles synthesized with OA, MA, and HA was studied to calculate the $\mathrm{HOMO}$ and the LUMO energy levels of the nanoparticles. CV experiments were performed on $\mathrm{PbS}$ nanoparticles dispersed in organic solution because a current needs to be generated through the electrode/solution interface by applying an external voltage, where a solid theoretical framework exists. ${ }^{36-38}$ It was decided not to perform the electrochemical studies of the synthesized $\mathrm{PbS}$ nanoparticles in the form of thin films deposited on top of the working electrode because of the known limitations of analyzing nanoparticulate thin films by electrochemical methods; among the limitations, we can mention the following: ${ }^{39}$ (1) Thin films made out of semiconductor nanoparticles are substantially consumed in the very first scan because of the fact that the electron transfer followed by the chemical reaction degrades the nanoparticles. ${ }^{40}$ (2) In thin films, nanoparticles can lose their identity because of the strong interaction between them, and also, the appearance of phenomena such as grain boundary and electrolyte diffusion can mask the electrochemical behavior of individual nanoparticles. On the other hand, in films, the measured current is not controlled by the particle diffusion; therefore, detailed analysis of such data is often complicated. Allowing the nanoparticles to diffuse would resolve this problem and facilitate the analysis. (3) In theoretical models, ${ }^{41}$ a condition 

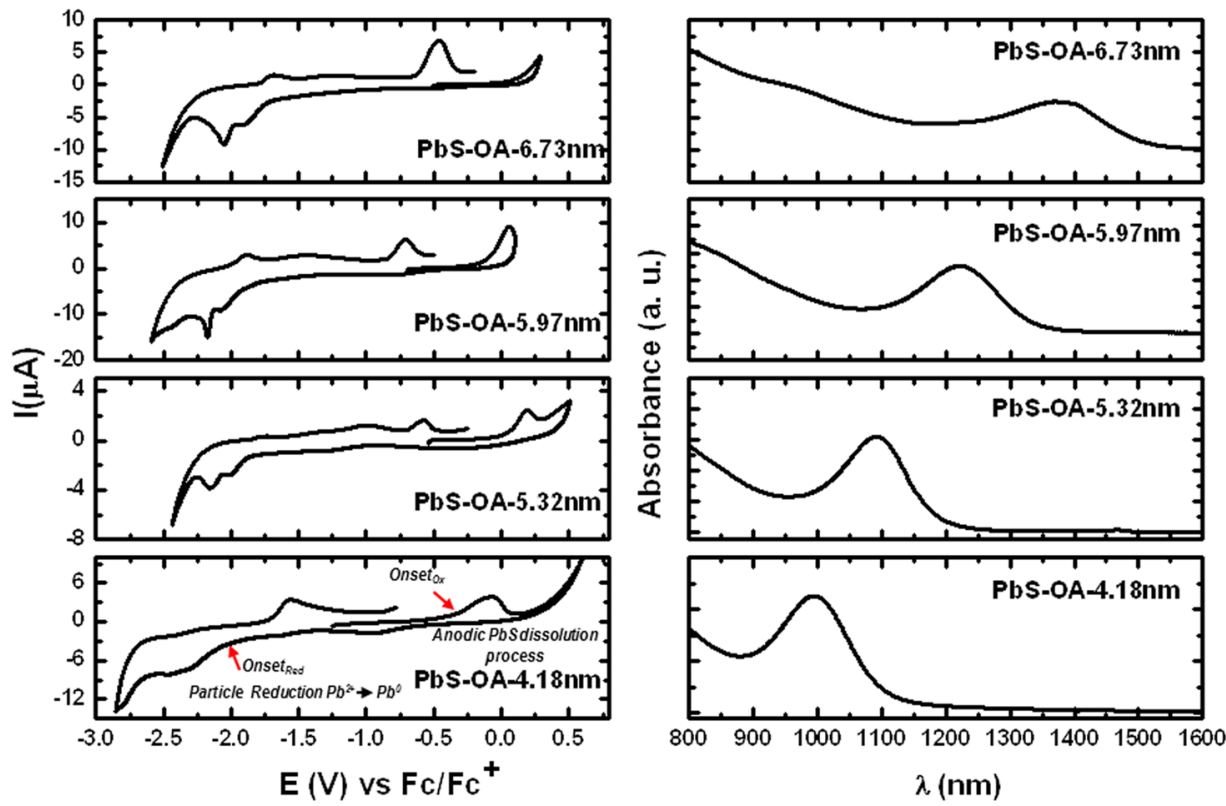

Figure 4. Left column: the voltammograms corresponding to the first scanning potential cycle for the OA-capped PbS nanoparticles. Right column: the UV-vis-NIR absorption spectra for each corresponding sample.
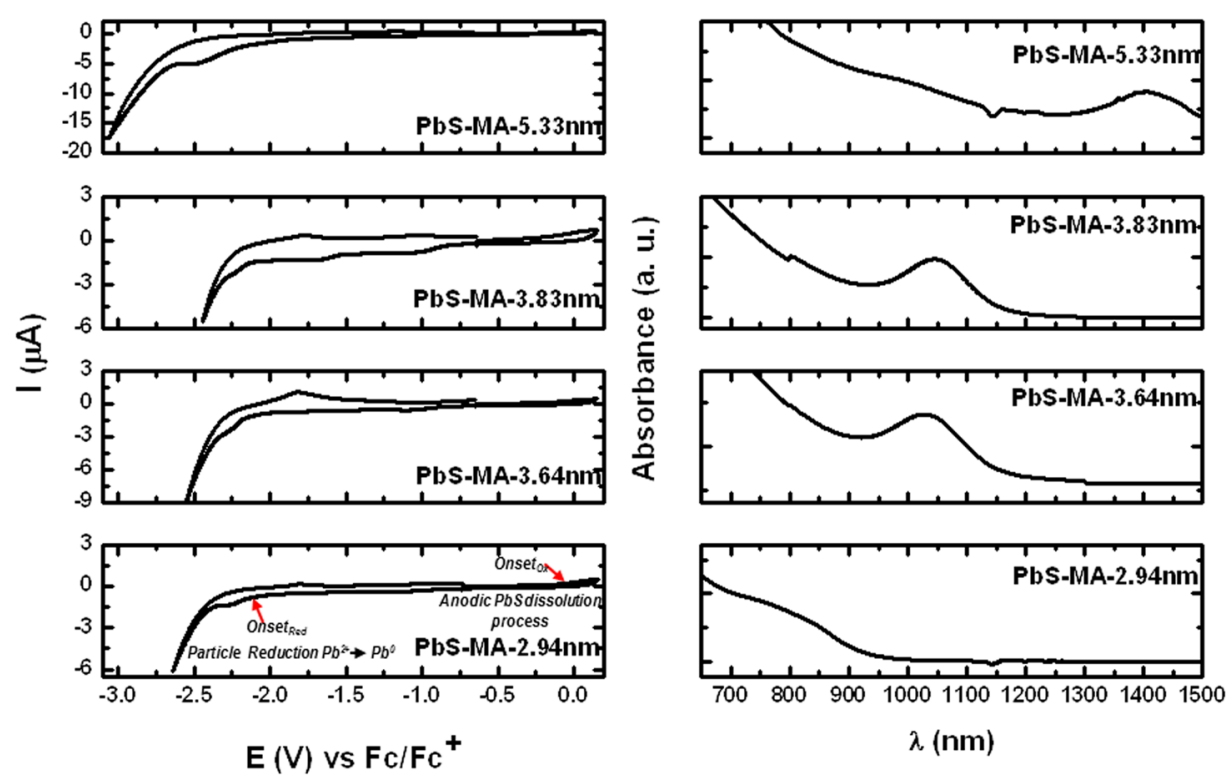

Figure 5. Left column: the voltammograms corresponding to the first scanning potential cycle for the MA-capped PbS nanoparticles. Right column: the UV-vis-NIR absorption spectra for each corresponding sample.

of "isolated nanoparticle" is assumed for the charge transfer from/to nanoparticles; this boundary condition is not satisfied in nanoparticle thin films because of the proximity to the electrode.

Figures 4-6 show the voltammograms and corresponding UV-vis-NIR absorbance spectra for the three synthesized PbS nanoparticle systems. The presented voltammograms are arranged by size, from largest to smallest, showing only the first cycle acquired with a clean Pt electrode, and the potential sweep starts toward oxidation potentials. To the right of each voltammogram, the corresponding UV-vis-NIR absorbance spectrum of the same sample is shown, where manifest quantum confinement effects can be observed for these systems, and the optical transition maximum wavelengths $\left(\lambda_{\max }\right)$ are summarized in Table 2 . All shown voltammograms in
Figures 4-6 displayed a diffusional redox response between 0.25 and $-2.5 \mathrm{~V}$ versus $\mathrm{Fc} / \mathrm{Fc}^{+}{ }^{29}$ The $\mathrm{CV}$ results for the three different nanoparticle systems studied show similar features: an oxidation signal can be observed between $\sim 0.0$ and $0.3 \mathrm{~V}$; meanwhile in the cathodic potential scanning, reduction processes can be observed between $\sim-1.7$ and $-2.2 \mathrm{~V}$. Then, after this reduction takes place and the potential scan is back to the anodic direction, only in the case of the OA-capped nanoparticles can be clearly observed a new anodic peak in the range between -0.5 and $-0.7 \mathrm{~V}$ (see Figures $4-6$ ). This is an indication that the reduced $\mathrm{PbS}$ nanoparticles have coupled chemical reactions that produce intermediary species, which is more evidently observed on the OA nanoparticles than on the MA and HA systems. 

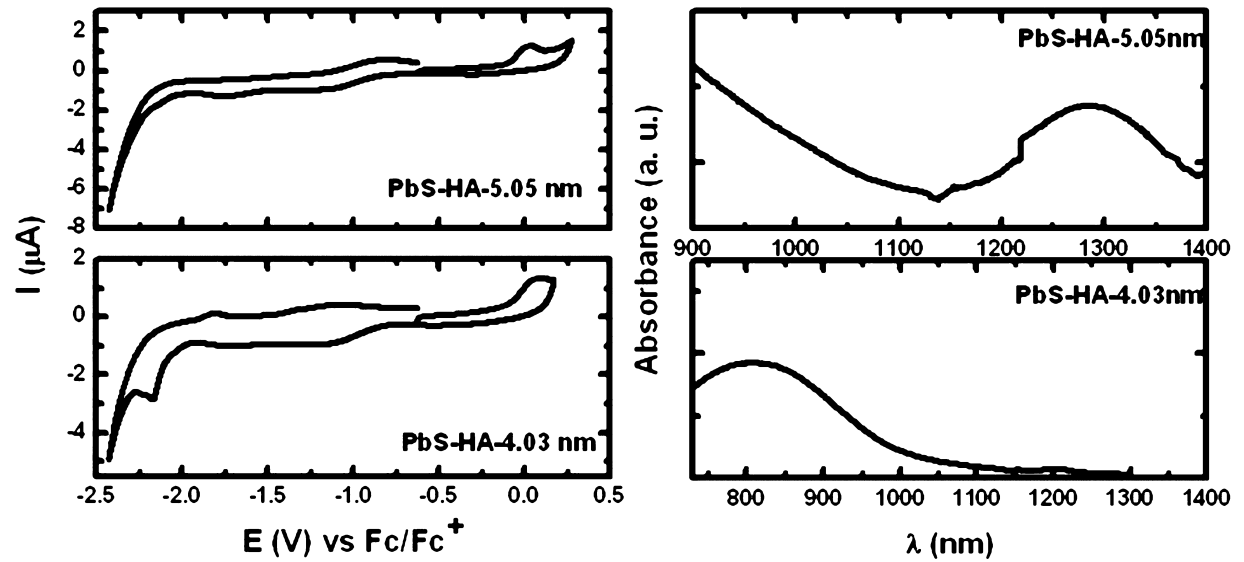

Figure 6. Left column: the voltammograms corresponding to the first scanning potential cycle for the HA-capped PbS nanoparticles. Right column: the UV-vis-NIR absorption spectra for each corresponding sample.

Table 2. Summary of the Results from the Characterization of the PbS Nanoparticles Synthesized with Different Capping Ligands $^{a}$

\begin{tabular}{|c|c|c|c|c|c|c|c|c|c|}
\hline system & size $(\mathrm{nm})$ & onset $_{\text {red }}(\mathrm{V})$ & onset $_{\text {oxi }}(\mathrm{V})$ & $E_{\mathrm{g}}^{\text {echem }}(\mathrm{eV})$ & Abs $\lambda_{\max }(\mathrm{nm})$ & $E_{\mathrm{g}}^{\mathrm{opt}}(\mathrm{eV})$ & $E_{\mathrm{g}}(\mathrm{EELS})(\mathrm{eV})$ & CB LUMO (eV) & VB HOMO $(\mathrm{eV})$ \\
\hline \multirow[t]{5}{*}{$\mathrm{PbS}-\mathrm{OA}$} & 4.18 & -2.04 & -0.30 & 1.76 & 1000 & 1.11 & 1.35 & 3.06 & 4.24 \\
\hline & 5.32 & -1.87 & 0.05 & 1.92 & 1095 & 1.07 & & 3.23 & 4.36 \\
\hline & 5.97 & -1.86 & -0.11 & 1.75 & 1222 & 0.95 & & 3.24 & 4.24 \\
\hline & 6.27 & -1.88 & -0.11 & 1.77 & 1285 & 0.89 & & 3.22 & 4.16 \\
\hline & 6.73 & -1.71 & 0.12 & 1.83 & 1368 & 0.842 & & 3.39 & 4.28 \\
\hline \multirow[t]{5}{*}{ PbS-MA } & 2.94 & -2.03 & -0.10 & 1.93 & 779 & 1.30 & 1.5 & 3.07 & 4.47 \\
\hline & 3.64 & -2.11 & -0.16 & 1.95 & 1029 & 1.046 & & 2.99 & 4.12 \\
\hline & 3.83 & -2.13 & -0.19 & 1.94 & 1049 & 1.054 & & 2.97 & 4.10 \\
\hline & 4.08 & -2.14 & -0.08 & 2.06 & 1166 & 0.96 & & 2.96 & 3.99 \\
\hline & 5.33 & -2.24 & -0.13 & 2.11 & 1400 & 0.79 & & 2.86 & 3.71 \\
\hline \multirow[t]{2}{*}{$\mathrm{PbS}-\mathrm{HA}$} & 4.03 & -2.07 & -0.12 & 1.95 & 817 & 1.20 & & 3.03 & 4.30 \\
\hline & 5.05 & -2.08 & -0.10 & 1.98 & 1285 & 0.80 & & 3.02 & 3.88 \\
\hline
\end{tabular}

${ }^{a}$ Reduction and oxidation onsets with respect to ferrocene $(\mathrm{Fc})$ used as an internal standard. $E_{\mathrm{g}}^{\text {echem }}=$ electrochemical band gap, Abs = maximum value of the first optical absorption peak, $E_{\mathrm{g}}^{\text {opt }}=$ optical band gap, and $E_{\mathrm{g}}(\mathrm{EELS})=$ band gap calculated through EELS. HOMO and LUMO are calculated on an absolute energy scale using the following equations: LUMO $=-\left(\right.$ onset $\left._{\text {red }}+5.1\right)(\mathrm{eV})(3)^{20}$ and $\mathrm{HOMO}=\left[\mathrm{LUMO}-E_{\mathrm{g}}^{\mathrm{opt}}-1.786 \frac{e^{2}}{4 \pi \varepsilon_{0} \varepsilon_{\mathrm{QD}} R}\right](\mathrm{eV})(4)^{10}$

The redox behavior of the dispersed $\mathrm{PbS}$ nanoparticles in the electrolyte to the applied voltage stimulus shows a similarity to previous reports. ${ }^{42-44}$ The observed anodic process can be associated with the sulfur oxidation (anodic dissolution, eq 1), whereas the reduction process can be associated with the lead reduction (eq 2). On the other hand, the current wave measured at -0.5 and $-0.7 \mathrm{~V}$, obtained after the nanoparticle reduction and clearly observed for the PbS-OA system (see Figure 4), can be related to the metallic lead deposition on the electrode surface, which is reoxidized when the potential scanning reaches positive potentials (eq 2). These main processes are indicated in Figures 4-6.

$$
\begin{array}{ll}
\text { Anodic dissolution }^{43} & \mathrm{PbS} \rightarrow \mathrm{Pb}^{2+}+\mathrm{S}+2 \mathrm{e}^{-} \\
\text {Cathodic reduction }^{43} & \mathrm{PbS}+2 \mathrm{e}^{-} \rightarrow \mathrm{Pb}+\mathrm{S}^{2-}
\end{array}
$$

According to the reviewed refs $42-44$ and the analyses of the obtained voltammograms, it is concluded that the two observed defined redox processes can be reliably related to the PbS nanoparticles. These anodic and cathodic redox processes are separated by a planar region where no voltammetric features can be observed. The physical separation between the oxidation and reduction onsets is related to an electrochemical band gap $\left(E_{\mathrm{g}}^{\mathrm{echem}}\right)$, and the values obtained for the three studied systems are summarized in Table 2, along with other electrochemical and optical parameters. ${ }^{17}$ It is observed that all three different systems show similar behaviors and features in their voltammetry response. However, the current signal related to the $\mathrm{PbS}$ nanoparticle oxidation (the anodic dissolution process) can be sensitive to the capping ligand nature; for example, $\mathrm{PbS}$ MA, in Figure 5, shows that the oxidation process is suppressed with regard to $\mathrm{PbS}-\mathrm{HA}$ and $\mathrm{PbS}-\mathrm{OA}$. Also, the oxidation of electrodeposited lead, after $\mathrm{PbS}$ nanoparticle reduction eq 2, decreases noticeably for the MA and HA systems (see Figures 4-6). Therefore, this shows that the capping ligand can influence the electrochemical behavior of the nanoparticles. It seems that in the cases of MA and HA, the anodic dissolution is stabilized and avoids the lead electrodeposition reaction. The chemical reactions associated with the electrochemical processes affect the redox process reversibility, and this can mask the size confinement effect on the redox onset potentials; care should be taken when the electrochemical parameters for the three studied systems are analyzed.

A potential onset is defined as the potential where the injection of electrons or holes toward the LUMO and HOMO levels, respectively, becomes evident by an increment in the cathodic or anodic current. ${ }^{29}$ 
Small variations in the reduction onset value can be observed as the size changes for the OA and MA systems, whereas for the HA system, only two different samples displayed results of enough quality to be analyzed, and no clear differences could be observed between them. The variations observed in the reduction onset values of the OA and MA systems could be related to the quantum confinement effects displayed by the nanoparticles. The mentioned variation was observed to be opposite in those two systems: whereas in the OA system, the value of the reduction onset increased (became less negative) with increasing nanoparticle size, in the MA system, the value of the reduction onset decreased (became more negative) with increasing nanoparticle size. This trend is more evident when the largest and smallest nanoparticle sizes are compared because the reduction onset values for the other nanoparticle sizes in between are very close to each other.

Further studies trying to elucidate the energy levels of the synthesized $\mathrm{PbS}$ nanoparticles include EELS experiments within TEM. EELS provides information about the inelastic scattering events the electrons in the microscope beam undergo while traveling through the sample. Among these inelastic events we find the intra- and interband transitions, being the transition from the VB (HOMO) to the conduction band (CB) (LUMO) one of the most important in semiconductors; reason why this technique has been previously used to determine the band gap, $E_{g}$, of certain semiconductor materials. ${ }^{45,46}$ Figure S6 shows the acquired and treated spectra for samples PbS-MA$2.94 \mathrm{~nm}$ and $\mathrm{PbS}-\mathrm{OA}-4.18 \mathrm{~nm}$. It can be noticed that the optical band gap, $E_{\mathrm{g}}^{\text {opt }}$, is lower than the measured $E_{\mathrm{g}}$, for both samples. ${ }^{45-48^{8}}$ Sample PbS-MA-2.94nm showed an $E_{\mathrm{g}}$ of approximately $1.5 \mathrm{eV}$, whereas it displayed an $E_{\mathrm{g}}^{\text {opt }}$ of approximately $1.3 \mathrm{eV}$. On the other hand, sample $\mathrm{PbS}-\mathrm{OA}$ $4.18 \mathrm{~nm}$ showed an $E_{\mathrm{g}}$ of approximately $1.35 \mathrm{eV}$, whereas this same sample displayed an $E_{\mathrm{g}}^{\text {opt }}$ of approximately $1.1 \mathrm{eV}$. In both cases, $E_{\mathrm{g}}$ calculated through EELS was approximately $0.2 \mathrm{eV}$ higher than $E_{\mathrm{g}}^{\text {opt }}$. This difference between $E_{\mathrm{g}}^{\text {opt }}$ and $E_{\mathrm{g}}$ calculated using EELS has been previously attributed to the size distribution of the analyzed nanoparticles and the facts that $E_{\mathrm{g}}^{\text {opt }}$ gives average information of many nanoparticles analyzed at the same time, whereas $E_{\mathrm{g}}$ through EELS provides information of only a few nanoparticles. ${ }^{46}$

In Table 2, all data gathered from CV, UV-vis-NIR, and EELS are presented, whereas Figure 7 shows a graphical representation of the different energy levels calculated for the different nanoparticles. It is organized for the different nanoparticle systems (capping) by size. The first column

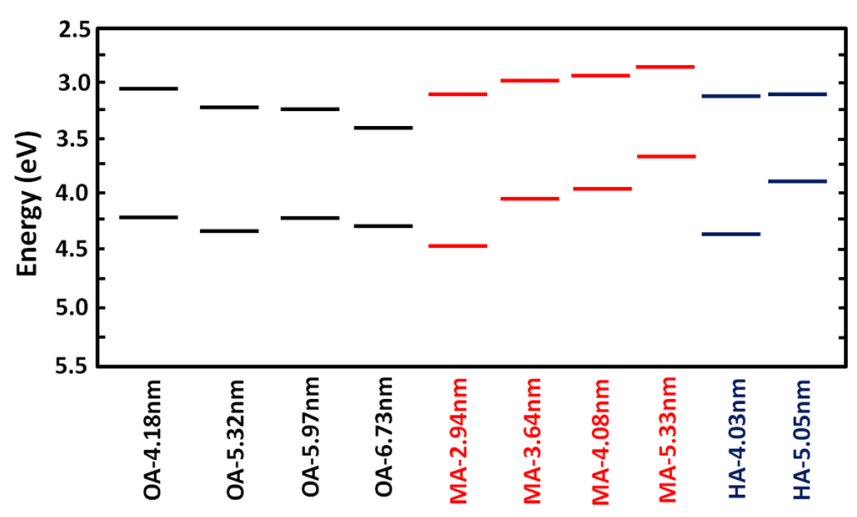

Figure 7. Graphic representation indicating the calculated energy levels for all different $\mathrm{PbS}$ nanoparticle systems analyzed. corresponds to the specific nanoparticle system; the second column shows the average sizes of the nanoparticles; and the following three columns show the relevant information acquired from the $\mathrm{CV}$ measurements, such as the oxidation and reduction onsets and the electrochemical band gaps.

In the following column can be found the values of the optical absorption peak or $\lambda_{\max }$ in $\mathrm{nm}$, and next to it is displayed $E_{\mathrm{g}}^{\text {opt}}$, calculated based on ref 49 . As previously mentioned, $E_{\mathrm{g}}^{\text {opt }}$ shows a well-defined quantum confinement effect. The next column shows the $E_{\mathrm{g}}$ (EELS) values calculated for samples $\mathrm{PbS}$ $\mathrm{OA}-4.18 \mathrm{~nm}$ and PbS-MA-2.94nm, based on the methodology previously described. ${ }^{46}$ Finally, the last two columns show the values calculated for the LUMO and HOMO based on eqs 3 and 4 in the footnote of Table 2.

The LUMO value is related to the electron affinity, which is the energy required to add an electron to an atom or a molecule in the gas phase. ${ }^{29}$ In this study, two electrochemical processes can be observed, as previously mentioned, a cathodic and an anodic peak. The cathodic peak $(\sim 1.6-2.2 \mathrm{~V})$ represents the reduction or the process through which electrons are adhering to the nanoparticles. Additionally, this reduction potential displays dependence on the nanoparticle size and capping ligand. Because the other important signal, the anodic peak, did not show evident variations with the size and is affected by the capping ligand, it was decided to use the reduction onset value to calculate the LUMO level of the nanoparticles. The LUMO level is calculated by adding $5.1 \mathrm{eV}$ to the reduction onset to change the LUMO level to an absolute energy scale, according to ref 29. The HOMO level was calculated based on the methodology suggested in ref 10 , where a Coulombic stabilization energy is considered between the electron-hole pair, due to the exciton generation, as shown in eq 4 in the footnote of Table 2 , where $e$ is the electron charge, $\varepsilon_{0}$ is the vacuum permittivity, $\varepsilon_{\mathrm{QD}}$ is the optical dielectric constant of the nanoparticles, and $R$ is the nanoparticle radius. ${ }^{10}$ Using these equations, the HOMO and LUMO values shown in Table 2 were calculated.

Figure 8 shows the CV curves and UV-vis-NIR absorbance results for samples PbS-HA-4.03nm, PbS-MA-4.08nm, and $\mathrm{PbS}-\mathrm{OA}-4.18 \mathrm{~nm}$. In this case, these $\mathrm{PbS}$ nanoparticles display a similar average size of approximately $4 \mathrm{~nm}$, but with a different capping ligand. However, the calculated values for their $E_{\mathrm{g}}^{\mathrm{opt}}$ and $E_{\mathrm{g}}^{\text {echem }}$ show marked differences (see Table 2). Samples PbSOA-5.32nm and PbS-MA-5.33nm, not included in Figure 8, also showed a very similar size and size distribution, but with a difference of over $300 \mathrm{~nm}$ in the value of their optical absorption peak maxima. The observed effects of the capping ligand on the HOMO and LUMO values of $\mathrm{PbS}$ nanoparticles are in agreement with previous reports. ${ }^{10}$ Brown et al. attributed the differences in the HOMO and LUMO levels to the formation of dipoles on the surface of the nanoparticles due to the difference between the dielectric constants of the capping ligand and the nanoparticles and a contribution from the intrinsic dipole moment of the ligand itself, but assumed that the band gap remained constant, as the average size of the nanoparticles remained constant after the capping ligand exchange. $^{10}$ In the results presented in Figure 8 , it is undoubtedly shown that nanoparticles with a similar size but with different capping ligands (same anchoring group with different tail lengths) have different optical and electrochemical properties. These variations, in the absorption peak maxima and in the reduction onset values, observed in the same-sized nanoparticles translate into different calculated values of $E_{\mathrm{g}}^{\mathrm{opt}}$ 

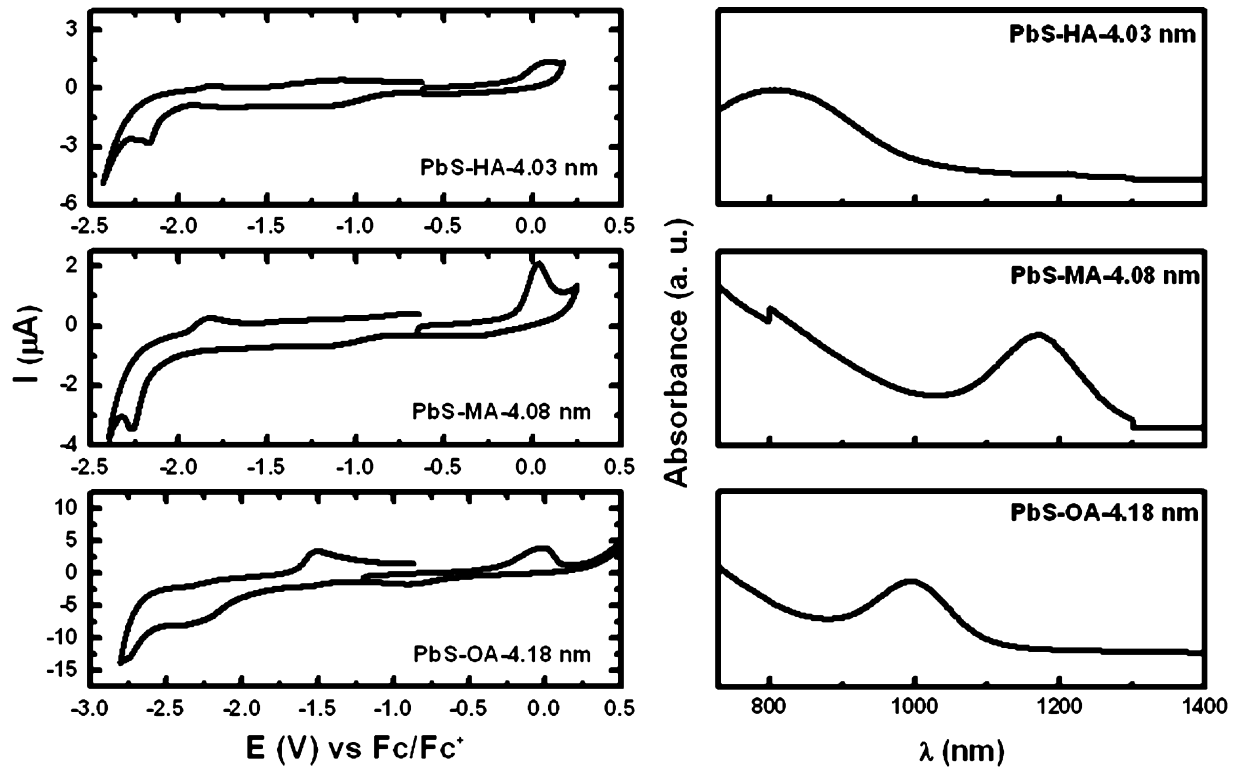

Figure 8. Voltammograms and UV-vis-NIR absorption spectra of PbS-HA-4.03nm, PbS-MA-4.08nm, and PbS-OA-4.18nm samples, all of them showing a nanoparticle average size of approximately $4 \mathrm{~nm}$.

and LUMO level. Even though the surface chemistries of the three systems are very similar, as evidenced by the FTIR and Raman results, and the dielectric constants of the three capping ligands are similar, the effect of changing the capping ligand found on the surface of the $\mathrm{PbS}$ nanoparticles on the values of the energy levels and the band gap is evident. As previously reported, ${ }^{10}$ the formation of dipoles on the nanoparticle surface, due to the variations in the dielectric constant of the capping layer, produces energy shifts in the levels of the nanoparticles; however, to affect the band gap, the ligand molecules should create new energy states within the band gap. Nonetheless, the used capping ligands (OA, MA, and HA) have the same anchoring functional groups $(-\mathrm{COO})$, which do not have orbital states located within $E_{\mathrm{g}}$ of the $\mathrm{PbS}$ nanoparticles, ${ }^{50,51}$ and even though they would, the new density of states should be high enough compared to $\mathrm{Pb}$ and $\mathrm{S}$ orbitals corresponding to intrinsic LUMO and HOMO levels, respectively. In this case, it seems inadequate to conceive the capping ligands as mere dipoles on the nanoparticle surface. Debellis et al. ${ }^{15}$ recently reported the enhancement of the optical absorption of colloidal $\mathrm{PbS}$ quantum dots, and they proposed an increment in the optical active sites induced by the extent of $\pi$ conjugation of the ligands and by the presence of heteroatoms either as electron-donor substituents or as part of the system. ${ }^{15}$

The capability of the capping ligand to determine the atomistic arrangement in metallic nanoparticles has been previously reported for the case of metallic nanoparticles, such as $\mathrm{Pd} .{ }^{52}$ Corthey et al. $^{52}$ reported the role the capping ligand molecules play on the way the $\mathrm{Pd}$ atoms arrange themselves in nanoparticles. They observed that $\mathrm{Pd}$ atoms arrange in a less ordered way in thiolate-capped nanoparticles than in amine-capped nanoparticles. This effect on the arrangement was most important on the surface of the nanoparticles; however, under some conditions, this effect was observed throughout the nanoparticle.

For the case of $\mathrm{PbS}$ nanoparticles with $\mathrm{OA}$ as the capping molecule, a recent study by Zherebetskyy et al. ${ }^{51}$ revealed that there is a preferential coverage of (001) surfaces by OA because of a strong binding energy originated from the carboxyl functional group. They found that the carboxyl group relaxes on the (001) surface by forming a bidentate bridge between the $(\mathrm{Pb}-\mathrm{O}-)$ and the $(\mathrm{S} \cdots \mathrm{HO}-)$ with the $\mathrm{Pb}$ and $\mathrm{S}$ surface atoms. ${ }^{51}$ On the other hand, the interaction between the OA and the (111) surface is significantly different because these surfaces are polar and require charge compensation; ${ }^{51}$ thus, the surface needs to be reconstructed or passivated. However, because of the steric interactions between the "tails" of the lead oleate molecules, it is not possible to have a full coverage of these (111) surfaces only by this capping ligand, and additional $-\mathrm{OH}$ groups are needed to provide charge balance. Hence, the (111) surfaces in these $\mathrm{PbS}$ nanoparticles are covered partially by $-\mathrm{OH}$ groups because of the length of the lead oleate molecules. This particular capping layer promotes an atomistic arrangement on the surface of $\mathrm{PbS}$ nanoparticles that produces a certain ratio between (100) and (111) facets on the surface of the nanoparticles. As previously mentioned, the equilibrium core shape of a nanoparticle can be obtained through the Wulff construction ratio: ${ }^{26}$

$$
w=\frac{h_{h k l}}{h_{100}}
$$

where $h_{h k l}$ is the distance from the center of the crystal to the $\{h k l\}$ facet along the direction perpendicular to the surface. ${ }^{51}$ In their study, Zherebetskyy et al. ${ }^{51}$ observed how varying the capping ligand from $\mathrm{OA}$ to acetic anhydride (AA) changes the atomistic arrangement of the nanoparticle surface. Whereas the OA-capped nanoparticles displayed a Wulff ratio $\left(h_{111} / h_{100}\right)$ of approximately 0.9 , the AA-capped nanoparticles did not show the (111) facets; instead, these $\mathrm{PbS}$ nanoparticles showed irregular shapes with steplike structures, related to the (100) facets. $^{51}$

Figure 9 shows the high-resolution TEM (HRTEM) images of the $\mathrm{PbS}$ nanoparticles synthesized with the different capping ligands. The HRTEM images of the PbS-OA nanoparticles are shown in Figure 9a,b; in those images, clearly faceted nanoparticles of approximately $4 \mathrm{~nm}$ in size can be observed displaying the (100) and (111) facets with a Wulff ratio of 


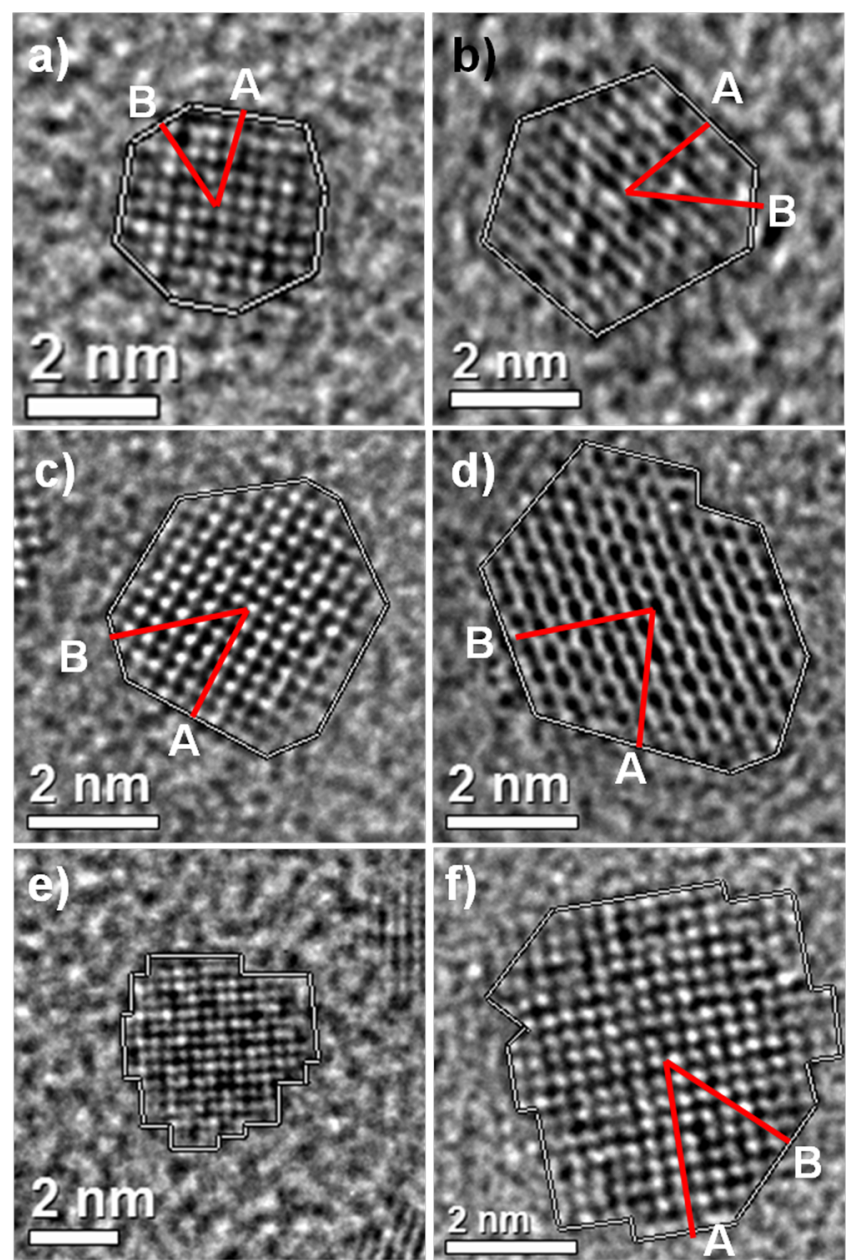

Figure 9. HRTEM images of (a,b) PbS-OA nanoparticles; (c,d) PbSHA nanoparticles; and (e,f) PbS-MA nanoparticles. Lines A and B correspond to the distance to the (100) and (111) facets, respectively, from the center of the nanoparticle.

approximately 1.1 for these nanoparticles. In Figure 9c,d, the HRTEM images of the PbS-HA nanoparticles can be observed; in this case, well-faceted nanoparticles displaying the (100) and (111) facets can be seen. However, the shape of the nanoparticles is more irregular compared to the PbS-OA case, with a Wulff ratio of approximately 1.09 for these nanoparticles. The HRTEM images of the PbS-MA nanoparticles are shown in Figure 9e,f; in this case, the nanoparticle shape is very irregular, compared to the previous cases. Even for the nanoparticles shown in Figure 9e, no clear (111) facets could be observed, and only the (100) facets could be seen; for the case of the nanoparticles shown in Figure 9f, where a few (111) facets were observed, a Wulff ratio of approximately 0.88 was calculated.

These images show the effect of the capping ligand molecule on the atomistic arrangement on the surface of $\mathrm{PbS}$ nanoparticles synthesized by the one-pot method; it can be observed that OA produces nanoparticles with more regular shapes than the other CAs, whereas MA produces nanoparticles with a more irregular shape. This irregular shape in the PbS-MA nanoparticles is associated with a difference in the atomistic arrangement on the nanoparticle surface. The Wulff ratio of 0.88 calculated from Figure 9f, the lowest of the three samples, can be associated with an increment in the presence of the (100) facets on the surface of the nanoparticles, compared to the (111) facets. It has been reported in the literature that increasing the presence of the (100) facets in $\mathrm{PbS}$ nanoparticles reduces the $\mathrm{Pb} / \mathrm{S}$ composition ratio, ${ }^{27}$ as this is confirmed in the data presented in Table 1. On the other hand, the Wulff ratio of 1.1 calculated for the PbS-OA nanoparticles, the highest of the three samples, can be associated with an increment in the presence of the (111) facets on the surface of the nanoparticles, compared to the (100) facets. As the (111) facets are $\mathrm{Pb}$ terminated, ${ }^{23}$ an increment in the presence of these facets on the surface of $\mathrm{PbS}$ nanoparticles will increase the $\mathrm{Pb} / \mathrm{S}$ ratio, as this can be confirmed in Table 1 .

The previous discussion shows the effect of the capping ligand on the final shape of the synthesized $\mathrm{PbS}$ nanoparticles, particularly on their atomistic surface arrangement, that translates into which crystallographic facets are present on their surface. Nonetheless, a correlation between this atomistic surface arrangement and the value of the band gap has not been established. As it has been previously mentioned, the variations observed in the band gap cannot be attributed to the orbital states of ligand molecules located within $E_{\mathrm{g}}$ of the $\mathrm{PbS}$ nanoparticles ${ }^{50,51}$ but are related to the confinement effects involving preferred atomic facets found on the surface of the nanoparticles. From a molecular point of view, as a molecule becomes larger, the number of atomic orbitals that overlap to form molecular orbitals increases, leading to an increasingly larger number of energy levels, thus decreasing the HOMOLUMO energy gap. Each molecular orbital has a well-defined energy value, located at intermediate values of the energy gap. For several hundred atoms, these intermediate energy levels, closely spaced to each other, end up forming a continuum band. Clearly, this molecular explanation provides a simple understanding on the size dependence of the HOMO-LUMO energy gap; however, this is valid only for an increasingly larger number of identical orbitals, with the same energy and symmetry. ${ }^{53}$ For PbS nanoparticles, it is a little more complicated, due essentially to two factors: (1) $\mathrm{Pb}$ and $\mathrm{S}$ are elements with different electronegativities and different types of atomic orbitals are combined and (2) the ratio between core/ surface molecular states.

First, the electronic configuration of $\mathrm{S}$ and $\mathrm{Pb}$ are $[\mathrm{Ne}] 3 \mathrm{~s}^{2}$ $3 \mathrm{p}^{4}$ and $[\mathrm{Xe}] 4 \mathrm{f}^{14} 5 \mathrm{~d}^{10} 6 \mathrm{~s}^{2} 6 \mathrm{p}^{2}$, respectively. From a linear combination of atomic orbital approach and from a hypothetical $\mathrm{PbS}$ single unit, the HOMO will be closer in energy to the $3 \mathrm{p}(\mathrm{S})$ and $6 \mathrm{~s}(\mathrm{~Pb})$ orbitals, whereas the LUMO will be closer in energy to the $3 \mathrm{~s}(\mathrm{~S})$ and $6 \mathrm{p}(\mathrm{Pb})$ orbitals. ${ }^{54}$ As an increasingly larger number of units are combined in an hypothetical $1 \mathrm{D} \mathrm{PbS}$ crystal, the VB would be progressively constructed from a linear combination of $3 \mathrm{p}(\mathrm{S})$ and $6 \mathrm{~s}(\mathrm{~Pb})$ orbitals, whereas the $\mathrm{CB}$ would be formed from $3 \mathrm{~s}(\mathrm{~S})$ and $6 \mathrm{p}(\mathrm{Pb})$ orbitals. ${ }^{54}$ Edges of these bands would be primarily constituted by most bonding and antibonding molecular orbitals. Again, from the previous molecular model, for a few hundred atoms, these closely degenerated states would constitute the HOMO and LUMO extended bands; as the size increases, the band gap separating them would reduce. It would be expected that as these $\mathrm{PbS} 1 \mathrm{D}$ chains keep growing in all directions, eventually they will reach a size that will display a band gap corresponding to the value of bulk $\mathrm{PbS}$.

Second, for $\mathrm{PbS}$ nanoparticles with a size ranging from 2.94 to $6.73 \mathrm{~nm}$, contributions of molecular orbitals of $\mathrm{PbS}$ units from the surface and the core of the nanoparticles are of the same order of magnitude. Thus, shapes and crystallographic preferred facets found on the surface would change this core/ 
surface ratio. Recently, it has been demonstrated that for $\mathrm{H}$ terminated nanodiamonds, the HOMO at the top of the VB is dominated by the contribution from carbon atoms in the core, whereas the LUMO at the base of the CB is dominated by the contribution from carbon atoms on the surface. ${ }^{55}$ Also, in a similar study, Barnard et al. ${ }^{56}$ were able to observe a shapedependent trend in the electronic properties of diamond nanoparticles. They studied 17 nanoparticles, ranging from 259 $\mathrm{C}$ atoms $(\sim 1.2 \mathrm{~nm})$ to $1798 \mathrm{C}$ atoms $(\sim 3.3 \mathrm{~nm})$, including entirely (111), majority (111), majority (100), and majority (110) facets on their surface. Their results showed a variation in the energy value of the LUMO level for entirely (111) and (110), inversely proportional to the size, whereas shapes containing a combination of (111) and (100) facets showed a different trend. ${ }^{56}$ Undoubtedly, a similar shape dependency in the final energy values of the HOMO and LUMO levels can be observed in $\mathrm{PbS}$ nanoparticles in the results shown in Figures 7 and 9. The $\mathrm{PbS}-\mathrm{OA}$ and $\mathrm{PbS}-\mathrm{HA}$ nanoparticles show a predominant size dependence of energy gap, whereas in the PbS-MA nanoparticles, this trend is lost (Figure 7). On the other hand, PbS-OA and PbS-HA exhibit similar Wulff ratios $(\sim 1.1)$, which can be interpreted to represent a similar presence of (111) and (100) facets on the surface of these nanoparticles, increasing the contribution of surface molecular orbitals due to the presence of the (111) higher atomic density facets on the surface, whereas in the PbS-MA nanoparticles, a majority of (100) facets are present on the surface, inverting the size dependence trend, an observation that could be related to the fact that the (100) facets found on the surface of these nanoparticles are lower atomic density planes, compared to the (111) facets, thus reducing the amount of atoms on the surface and increasing the contribution of the core molecular orbitals of the nanoparticle. Thus, it seems that OA and HA passivation guarantees a similar presence of the (111) and (100) facets on the surface of the $\mathrm{PbS}$ nanoparticles and a quantum confinement control related mostly to the size. A detailed theoretical simulation study is underway trying to gain a better understanding on the effect the surface atomistic arrangement in $\mathrm{PbS}$ nanoparticles has on the values of their energy band gap and HOMO and LUMO levels.

Another effect related to the variations in the type of facets found on the nanoparticle surface is the appearance of additional phononic vibrations. It is clear that as the presence of the (100) facets on the surface of the nanoparticles increases, a band starts to appear on the Raman spectrum (Figure 3) at approximately $61 \mathrm{~cm}^{-1}$, as it is clearly observed in the PbS-MA sample (a Wulff ratio of 0.88 ). This band could be attributed to a phononic vibration related to a nanoparticle surface with a lower density of atoms because the (100) facets in fcc structures show a lower atomic density compared to the (111) facets. As the presence of the (111) facets on the nanoparticle surface starts to increase, the intensity of this band at approximately $61 \mathrm{~cm}^{-1}$ starts to decrease, as observed for the cases of the PbS-MA and PbS-OA samples, with Wulff ratios of $\sim 1.09$ and $\sim 1.1$, respectively. It has been recently reported that nanoparticles of semiconductor materials display a softening of their surface, ${ }^{34}$ which is strongly related to the presence of the capping molecules. Bozyigit et al. $^{34}$ concluded that this mechanical softening of the nanoparticle surface enabled the appearance of a large number ( $>100)$ of low-frequency phonon modes that can efficiently drive multiphonon processes. They also proposed that phonon interactions, including the scattering with electrons, neutrons, photons, and other phonons, will be enhanced in nanomaterials because of this surface softening and should be taken more importantly into consideration. Further studies are underway trying to gain a more complete understanding on the origin of this band on the Raman spectrum of $\mathrm{PbS}$ nanoparticles at approximately $61 \mathrm{~cm}^{-1}$.

These results are evidence that the value of the absorption peak maximum in $\mathrm{PbS}$ nanoparticles is related not only to the size of the nanoparticles, due to the quantum confinement effects, but also to the atomistic arrangement of the nanoparticle surface, which is determined by the nature of the capping ligand molecule. Further studies, both theoretical and experimental, are needed to get a more complete understanding of the relationship between the values of the energy band gap, the HOMO and LUMO values, and the atomistic arrangement of the nanoparticle surface.

\section{CONCLUSIONS}

$\mathrm{PbS}$ nanoparticles synthesized with different capping ligands, in particular, OA, MA, and HA, avoiding the common ligand exchange process, displayed an fcc structure and a similar composition, regardless of the CA used in the synthesis, with slight variations in the $\mathrm{Pb} / \mathrm{S}$ ratio, being highest for the case of $\mathrm{OA}$ and lowest for MA. The main difference observed between the three different capping ligands was the average size and the size distribution. These differences in the average size and size distribution of the nanoparticles were clearly observed in their UV-vis-NIR spectra, where all nanoparticle systems displayed quantum confinement effects. FTIR and Raman studies showed a very similar surface chemistry for the nanoparticles with the different capping ligands, displaying only small variations in the energy associated with the excitation of a LA phonon mode associated with $\mathrm{PbS}$ nanoparticles, and the clear appearance of an additional band at approximately $61 \mathrm{~cm}^{-1}$ for the case of the PbS-MA nanoparticles. The electrochemical studies of nanoparticles show the typical behavior expected for $\mathrm{PbS}$, displaying an oxidation signal associated with $\mathrm{PbS}$ anodic dissolution and a reduction signal associated with the cathodic reduction of $\mathrm{Pb}^{2+}$ to $\mathrm{Pb}^{0}$. However, the electrochemical analysis provides evidence that differences in the ligand carbon chain length and morphology affect the chemical reactions associated with the charged $\mathrm{PbS}$ nanoparticles. With all of these data, a reliable sketch of the energy levels in $\mathrm{PbS}$ nanoparticles of different sizes and with different capping ligands can be drawn. Varying the capping ligand of the nanoparticles not only affects the position of the HOMO and LUMO energy levels but also affects the value of $E_{g}$, as it was observed for nanoparticles of a similar size but with different capping ligands. Whereas in the literature, it has been referenced that the variations in the value of $E_{\mathrm{g}}$ in semiconductor nanoparticles depend only on the size, through quantum confinement effects, the current work presents experimental evidence on how the atomistic arrangement on the nanoparticle surface, through the type of crystallographic facets present on this surface, determined by the nature of the capping ligand molecule, also has an effect on the value of $E_{\mathrm{g}}$ and the energy values of the HOMO and LUMO levels. Further theoretical and experimental studies are needed to gain a better understanding on the effect the surface atomistic arrangement in $\mathrm{PbS}$ nanoparticles has on the values of their energy band gap and HOMO and LUMO levels. 


\section{ASSOCIATED CONTENT}

\section{S Supporting Information}

The Supporting Information is available free of charge on the ACS Publications website at DOI: 10.1021/acsomega.7b01451.

TEM images of the nanoparticles PbS-HA, PbS-MA, and $\mathrm{PbS}-\mathrm{OA}$ at different magnifications, EDXS spectra and calculated composition for the samples PbS-HA and PbSMA, FTIR data for PbS-OA, PbS-HA, and PbS-MA, and EELS data acquired for the samples PbS-MA-2.94nm and PbS-OA-4.18nm (PDF)

\section{AUTHOR INFORMATION}

\section{Corresponding Author}

*E-mail: domingo.garciagt@uanl.edu.mx. Phone: +52 (81) 1340-4000 ext. 1525 (D.I.G.-G.).

\section{ORCID}

Fernando Fungo: 0000-0003-3291-0829

Domingo Ixcoatl García-Gutiérrez: 0000-0001-6350-2161

\section{Notes}

The authors declare no competing financial interest.

\section{ACKNOWLEDGMENTS}

This work was supported by the Mexican Secretary of Education (SEP), PROMEP program, through the project "Apoyo a la Incorporacion de Nuevos PTC", project number PROMEP/103.5/10/3889, and also by CONACYT through project numbers 148691 and 154303 . The authors thank the International Center for Nanotechnology and Advanced Materials, ICNAM, at the University of Texas at San Antonio, for the support provided for the use of their Kleberg Advanced Microscopy Center. D.F.G.-G. and L.P.H.-C. acknowledge the financial support received from CONACYT Mexico. We also thank Consejo Nacional de Investigaiones Cientificas y Tecnicas (CONICET-Argentina), Agencia Nacional de Promoción Científica y Tecnológica (ANPCYT Argentina), Universidad Nacional de Río Cuarto. F.F. is a scientific member of CONICET.

\section{REFERENCES}

(1) McDonald, S. A.; Konstantatos, G.; Zhang, S.; Cyr, P. W.; Klem, E. J. D.; Levina, L.; Sargent, E. H. Solution-processed PbS quantum dot infrared photodetectors and photovoltaics. Nat. Mater. 2005, 4, $138-142$.

(2) Konstantatos, G.; Sargent, E. H. PbS colloidal quantum dot photoconductive photodetectors: Transport, traps, and gain. Appl. Phys. Lett. 2007, 91, 173505.

(3) Szendrei, K.; Cordella, F.; Kovalenko, M. M.; Böberl, M.; Hesser, G.; Yarema, M.; Jarzab, D.; Mikhnenko, O. V.; Gocalinska, A.; Saba, M.; Quochi, F.; Mura, A.; Bongiovanni, G.; Blom, P. W. M.; Heiss, W.; Loi, M. A. Solution-Processable Near-IR Photodetectors Based on Electron Transfer from $\mathrm{PbS}$ Nanocrystals to Fullerene Derivatives. Adv. Mater. 2009, 21, 683-687.

(4) Zhao, N.; Osedach, T. P.; Chang, L.-Y.; Geyer, S. M.; Wanger, D.; Binda, M. T.; Arango, A. C.; Bawendi, M. G.; Bulovic, V. Colloidal PbS Quantum Dot Solar Cells with High Fill Factor. ACS Nano 2010, 4, 3743-3752.

(5) Szendrei, K.; Gomulya, W.; Yarema, M.; Heiss, W.; Loi, M. A. $\mathrm{PbS}$ nanocrystal solar cells with high efficiency and fill factor. Appl. Phys. Lett. 2010, 97, 203501.

(6) Chang, L.-Y.; Lunt, R. R.; Brown, P. R.; Bulović, V.; Bawendi, M. G. Low-Temperature Solution-Processed Solar Cells Based on PbS Colloidal Quantum Dot/CdS Heterojunctions. Nano Lett. 2013, 13, 994-999.
(7) Chuang, C.-H. M.; Brown, P. R.; Bulović, V.; Bawendi, M. G. Improved performance and stability in quantum dot solar cells through band alignment engineering. Nat. Mater. 2014, 13, 796-801.

(8) Ko, D.-K.; Maurano, A.; Suh, S. K.; Kim, D.; Hwang, G. W.; Grossman, J. C.; Bulović, V.; Bawendi, M. G. Photovoltaic Performance of PbS Quantum Dots Treated with Metal Salts. ACS Nano 2016, $10,3382-3388$.

(9) Cao, Y.; Stavrinadis, A.; Lasanta, T.; So, D.; Konstantatos, G. The role of surface passivation for efficient and photostable $\mathrm{PbS}$ quantum dot solar cells. Nat. Energy 2016, 1, 16035.

(10) Brown, P. R.; Kim, D.; Lunt, R. R.; Zhao, N.; Bawendi, M. G.; Grossman, J. C.; Bulović, V. Energy Level Modification in Lead Sulfide Quantum Dot Thin Films through Ligand Exchange. ACS Nano 2014, $8,5863-5872$.

(11) Jasieniak, J.; Califano, M.; Watkins, S. E. Size-Dependent Valence and Conduction Band-Edge Energies of Semiconductor Nanocrystals. ACS Nano 2011, 5, 5888-5902.

(12) Soreni-Harari, M.; Yaacobi-Gross, N.; Steiner, D.; Aharoni, A.; Banin, U.; Millo, O.; Tessler, N. Tuning Energetic Levels in Nanocrystal Quantum Dots through Surface Manipulations. Nano Lett. 2008, 8, 678-684.

(13) Hines, M. A.; Scholes, G. D. Colloidal PbS Nanocrystals with Size-Tunable Near-Infrared Emission: Observation of Post-Synthesis Self-Narrowing of the particle size Distribution. Adv. Mater. 2003, 15, 1844-1849.

(14) Heimel, G.; Romaner, L.; Zojer, E.; Brédas, J.-L. Toward Control of the Metal-Organic Interfacial Electronic Structure in Molecular Electronics: A First-Principles Study on Self-Assembled Monolayers of Pi-Conjugated Molecules on Noble Metals. Nano Lett. 2007, 7, 932-940.

(15) Debellis, D.; Gigli, G.; ten Brink, S.; Infante, I.; Giansante, C. Quantum-Confined and Enhanced Optical Absorption of Colloidal $\mathrm{PbS}$ Quantum Dots at Wavelengths with Expected Bulk Behavior. Nano Lett. 2017, 17, 1248-1254.

(16) Hyun, B.-R.; Zhong, Y.-W.; Bartnik, A. C.; Sun, L.; Abruña, H. D.; Wise, F. W.; Goodreau, J. D.; Matthews, J. R.; Leslie, T. M.; Borrelli, N. F. Electron Injection from Colloidal PbS Quantum Dots into Titanium Dioxide Nanoparticles. ACS Nano 2008, 2, 2206-2212.

(17) Garcia-Gutierrez, D. I.; De Leon-Covian, L. M.; GarciaGutierrez, D. F.; Treviño-Gonzalez, M.; Garza-Navarro, M. A.; Sepulveda-Guzman, $\mathrm{S}$. On the role of $\mathrm{Pb}^{0}$ atoms on the nucleation and growth of $\mathrm{PbSe}$ and $\mathrm{PbTe}$ nanoparticles. J. Nano Res. 2013, 15, 1620.

(18) Garcia-Gutierrez, D. I.; Garcia-Gutierrez, D. F.; De LeonCovian, L. M.; Treviño-Gonzalez, M. T.; Garza-Navarro, M. A.; Moreno-Cortez, I. E.; Cienfuegos-Pelaes, R. F. Aberration Corrected STEM Study of the Surface of Lead Chalcogenide Nanoparticles. J. Phys. Chem. C 2014, 118, 22291-22298.

(19) Zhou, Y.; Wang, F.; Buhro, W. E. Large Exciton Energy Shifts by Reversible Surface Exchange in 2D II-VI Nanocrystals. J. Am. Chem. Soc. 2015, 137, 15198-15208.

(20) Newton, J. C.; Ramasamy, K.; Mandal, M.; Joshi, G. K.; Kumbhar, A.; Sardar, R. Low-temperature synthesis of magic-sized CdSe nanoclusters: influence of ligands on nanocluster growth and photophysical properties. J. Phys. Chem. C 2012, 116, 4380-4389.

(21) Mahler, B.; Nadal, B.; Bouet, C.; Patriarche, G.; Dubertret, B. Core/shell colloidal semiconductor nanoplatelets. J. Am. Chem. Soc. 2012, 134, 18591-18598.

(22) Lawrence, K. N.; Johnson, M. A.; Dolai, S.; Kumbhar, A.; Sardar, R. Solvent-like ligand-coated ultrasmall cadmium selenide nanocrystals: strong electronic coupling in a self-organized assembly. Nanoscale 2015, 7, 11667-11677.

(23) Nevers, D. R.; Williamson, C. B.; Hanrath, T.; Robinson, R. D. Surface chemistry of cadmium sulfide magic-sized clusters: a window into ligand-nanoparticle interactions. Chem. Commun. 2017, 53, 28662869.

(24) Landes, C.; Braun, M.; Burda, C.; El-Sayed, M. A. Observation of Large Changes in the Band Gap Absorption Energy of Small CdSe 
Nanoparticles Induced by the Adsorption of a Strong Hole Acceptor. Nano Lett. 2001, 1, 667-670.

(25) Bealing, C. R.; Baumgardner, W. J.; Choi, J. J.; Hanrath, T.; Hennig, R. G. Predicting Nanocrystal Shape through Consideration of Surface-Ligand Interactions. ACS Nano 2012, 6, 2118-2127.

(26) Ringe, E.; Van Duyne, R. P.; Marks, L. D. Wulff Construction for Alloy Nanoparticles. Nano Lett. 2011, 11, 3399-3403.

(27) Choi, H.; Ko, J.-H.; Kim, Y. H.; Jeong, S. Steric-HindranceDriven Shape Transition in PbS Quantum Dots: Understanding SizeDependent Stability. J. Am. Chem. Soc. 2013, 135, 5278-5281.

(28) García-Gutiérrez, D. F.; Ovalle, D. G.; Casillas, L. P. H.; Fungo, F.; Gutiérrez, D. I. G. Efecto del cambio de agente protector en las propiedades ópticas y eléctricas de nanopartículas de sulfuro de plomo (PbS). Ingenierias 2014, 17, 6-16.

(29) Cardona, C. M.; Li, W.; Kaifer, A. E.; Stockdale, D.; Bazan, G. C. Electrochemical considerations for determining absolute frontier orbital energy levels of conjugated polymers for solar cell applications. Adv. Mater. 2011, 23, 2367-2371.

(30) Moreels, I.; Justo, Y.; De Geyter, B.; Haustraete, K.; Martins, J. C.; Hens, Z. Size-Tunable, Bright, and Stable PbS Quantum Dots: A Surface Chemistry Study. ACS Nano 2011, 5, 2004-2012.

(31) Sliem, M. A.; Chemseddine, A.; Bloeck, U.; Fischer, A. PbSe nanocrystal shape development: oriented attachment at mild conditions and microwave assisted growth of nanocubes. CrystEngComm 2011, 13, 483-488.

(32) Wu, N.; Fu, L.; Su, M.; Aslam, M.; Wong, K. C.; Dravid, V. P. Interaction of Fatty Acid Monolayers with Cobalt Nanoparticles. Nano Lett. 2004, 4, 383-386.

(33) Blackburn, J. L.; Chappell, H.; Luther, J. M.; Nozik, A. J.; Johnson, J. C. Correlation between Photooxidation and the Appearance of Raman Scattering Bands in Lead Chalcogenide Quantum Dots. J. Phys. Chem. Lett. 2011, 2, 599-603.

(34) Bozyigit, D.; Yazdani, N.; Yarema, M.; Yarema, O.; Lin, W. M. M.; Volk, S.; Vuttivorakulchai, K.; Luisier, M.; Juranyi, F.; Wood, V. Soft surfaces of nanomaterials enable strong phonon interactions. Nature 2016, 531, 618-622.

(35) Kudin, K. N.; Ozbas, B.; Schniepp, H. C.; Prud'homme, R. K.; Aksay, I. A.; Car, R. Raman Spectra of Graphite Oxide and Functionalized Graphene Sheets. Nano Lett. 2008, 8, 36-41.

(36) Bard, A. J.; Faulkner, L. R. Electrochemical Methods. Fundamentals and Applications, 2nd ed.; John Wiley \& Sons, Inc: New York, 2001.

(37) Zoski, C. G. Handbook of Electrochemistry, 1st ed.; Elsevier: Amsterdam, 2007.

(38) Murray, R. W. Nanoelectrochemistry: Metal Nanoparticles, Nanoelectrodes, and Nanopores Royce. Chem. Rev. 2008, 108, 26882720.

(39) Inamdar, S. N.; Ingole, P. P.; Haram, S. K. Determination of Band Structure Parameters and the Quasi-Particle Gap of CdSe Quantum Dots by Cyclic Voltammetry. ChemPhysChem 2008, 9, 2574-2579.

(40) Kucur, E.; Riegler, J.; Urban, G. A.; Nann, T. Determination of quantum confinement in CdSe nanocrystals by cyclic voltammetry. J. Chem. Phys. 2003, 119, 2333-2337.

(41) Wang, L.-W.; Zunger, A. Pseudopotential calculations of nanoscale CdSe quantum dots. Phys. Rev. B: Condens. Matter Mater. Phys. 1996, 53, 9579-9582.

(42) Ogawa, S.; Hu, K.; Fan, F.-R. F.; Bard, A. J. Photoelectrochemistry of Films of Quantum Size Lead Sulfide Particles Incorporated in Self-Assembled Monolayers on Gold. J. Phys. Chem. B 1997, 101, 5707-5711.

(43) Chen, S.; Truax, L. A.; Sommers, J. M. Alkanethiolate-Protected PbS Nanoclusters: Synthesis, Spectroscopic and Electrochemical Studies. Chem. Mater. 2000, 12, 3864-3870.

(44) Cuharuc, A. S.; Kulyuk, L. L.; Lascova, R. I.; Mitioglu, A. A.; Dikusar, A. I. Electrochemical Characterization of PbS Quantum Dots Capped with Oleic Acid and PbS Thin Films-a Comparative Study. Surf. Eng. Appl. Electrochem. 2012, 48, 193-211.
(45) Lazar, S.; Botton, G. A.; Wu, M.-Y.; Tichelaar, F. D.; Zandbergen, H. W. Materials science applications of HREELS in near edge structure analysis and low energy loss spectroscopy. Ultramicroscopy 2003, 96, 535-546.

(46) Erni, R.; Browning, N. D. Quantification of the size-dependent energy gap of individual CdSe quantum dots by valence electron energy-loss spectroscopy. Ultramicroscopy 2007, 107, 267-273.

(47) French, R. H.; Müllejans, H.; Jones, D. J. Optical Properties of Aluminum Oxide: Determined from Vacuum Ultraviolet and Electron Energy-Loss Spectroscopies. J. Am. Ceram. Soc. 1998, 81, 2549.

(48) Van Benthem, K.; French, R. H.; Sigle, W.; Elsässer, C.; Rühle, M. Valence electron energy loss study of Fe-doped $\mathrm{SrTiO}_{3}$ and a $\Sigma 13$ boundary: electronic structure and dispersion forces. Ultramicroscopy 2001, 86, 303.

(49) Dholakia, D. A.; Solanki, G. K.; Patel, S. G.; Agarwal, M. K. Optical Band Gap studies of Tungsten Sulphoselenide Single Crystals Grown by DVT Technique. Scientia Iranica 2003, 10, 373-382.

(50) Wang, C.; Zhou, G.; Liu, H.; Wu, J.; Qiu, Y.; Gu, B.-L.; Duan, W. Chemical Functionalization of Carbon Nanotubes by Carboxyl Groups on Stone-Wales Defects: A Density Functional Theory Study. J. Phys. Chem. B 2006, 110, 10266-10271.

(51) Zherebetskyy, D.; Scheele, M.; Zhang, Y.; Bronstein, N.; Thompson, C.; Britt, D.; Salmeron, M.; Alivisatos, P.; Wang, L.-W. Hydroxylation of the surface of $\mathrm{PbS}$ nanoparticles passivated with oleic acid. Science 2014, 344, 1380-1384.

(52) Corthey, G.; Olmos-Asar, J. A.; Casillas, G.; Mariscal, M. M.; Mejía-Rosales, S.; Azcárate, J. C.; Larios, E.; José-Yacamán, M.; Salvarezza, R. C.; Fonticelli, M. H. Influence of Capping on the Atomistic Arrangement in Palladium Nanoparticles at Room Temperature. J. Phys. Chem. C 2014, 118, 24641-24647.

(53) Koole, R.; Groeneveld, E.; Vanmaekelbergh, D.; Meijerink, A.; de Mello Donegá, C. Size Effects on Semiconductor Nanoparticles. In Nanoparticles; de Mello Donegá, C., Ed. ; Springer: Berlin, Heidelberg, 2014: pp 29-31.

(54) Walsh, A.; Watson, G. W. The origin of the stereochemically active $\mathrm{Pb}$ (II) lone pair: DFT calculations on $\mathrm{PbO}$ and $\mathrm{PbS}$. Jour. Sol. St. Chem. 2005, 178, 1422-1428.

(55) Drummond, N. D.; Williamson, A. J.; Needs, R. J.; Galli, G. Electron emission from diamondoids: a diffusion quantum Montecarlo study. Phys. Rev. Lett. 2005, 95, 096801.

(56) Barnard, A. S. Shape-Dependent confinement of the nanodiamond band gap. Cryst. Growth Des. 2009, 9, 4860-4863. 